\title{
Optical identification of radio-loud active galactic nuclei in the ROSAT-Green-Bank sample with SDSS spectroscopy
}

\author{
De-Liang Wang ${ }^{1,2}$, Jian-Guo Wang ${ }^{1,2}$ and Xiao-Bo Dong ${ }^{3}$ \\ 1 National Astronomical Observatories/Yunnan Observatory, Chinese Academy of Sciences, Kunming, \\ Yunnan, 650011, China; dlwang@mail.ynao.ac.cn \\ ${ }^{2}$ Graduate School of the Chinese Academy of Sciences, 19A Yuquan Road, Beijing 100039, China \\ ${ }^{3}$ Center for Astrophysics, University of Science and Technology of China, Hefei, 230026, China
}

\begin{abstract}
Results of extended and refined optical identification of 181 radio/X-ray sources in the RASS-Green Bank (RGB) catalog (Brinkmann et al. 1997) are presented which have been spectroscopically observed in the Sloan Digital Sky Survey (SDSS) DR5. The SDSS spectra of the optical counterparts are modeled in a careful and self-consistent way by incorporating the host galaxy's starlight. The optical emission line parameters are presented, which are derived accurately and reliably, along with the radio $1.4-5 \mathrm{GHz}$ spectral indices estimated using (non-simultaneous) archival data. For 72 sources, the identifications are presented for the first time. It is confirmed that the majority of strong radio/X-ray emitters are radio-loud active galactic nuclei (AGNs), particularly blazars. Taking advantage of the high spectral quality and resolution and our refined spectral modeling, we are able to disentangle narrow line radio galaxies (NLRGs), as vaguely termed in most previous identification work, into Seyfert II galaxies and LINERs (low-ionization nuclear emission regions), based on the standard emission line diagnostics. The NLRGs in the RGB sample, mostly belonging to 'weak line radio galaxies' (Tadhunter et al. 1998), are found have optical spectra consistent predominantly with LINERs, and only a small fraction with Seyfert II galaxies. A small number of LINERs have radio power as high as $10^{23}-10^{26} \mathrm{~W} \mathrm{~Hz}^{-1}$ at $5 \mathrm{GHz}$, being among the strongest radio emitting LINERs known so far. Two sources are identified with radio-loud narrow line Seyfert 1 galaxies (NLS1s), a class of rare objects. The presence is also confirmed of flat-spectrum radio quasars whose radio-optical-X-ray effective spectral indices are similar to those of High-energy peaked BL Lacs (HBLs), as suggested by Padovani et al. (2003), albeit it is yet a debate as to whether this is the case for their actual spectral energy distributions.
\end{abstract}

Key words: galaxies: active; galaxies: quasars; BL Lacerate objects: general; X-rays: general; radio continuum: general.

\section{INTRODUCTION}

Sources detected in both X-ray and radio surveys are predominantly radio emitting active galactic nuclei (AGNs), especially blazars. Optical identification of such sources has proved to be an efficient way to compile radio-loud AGNs and blazar samples. With the advent of the ROSAT satellite-both the ROSAT all-sky survey (RASS, Voges et al. 1999) and pointed observations-in the X-ray band and several large area sky surveys in the radio bands, large samples of radio-loud AGNs and blazars have been discovered and identified. Examples of such samples are the RGB (RASS-Green Bank) sample comprising 2127 sources (Brinkmann et al. 1995, 1997, Laurent-Muehleisen et al. 1998, 1999), the REX (radio-emitting X-ray) sample (Caccianiga et al. 1999, 2000), the DXRBS (Deep X-ray radio blazar survey) sample (Perlman 
et al. 1998, Landt et al.2001), the "Sedentary" sample (Giommi et al. 1999, 2005, Piranomonte et al. 2007), and the ROXA sample (Turriziani et al. 2007), etc., which were compiled using various selection criteria. Objects identified optically in these samples include radio quasars, BL Lac objects, broad-line and narrowline radio galaxies, and even clusters of galaxies. For most of these samples, the optical identification is far from complete; more identifications still await new optical spectroscopic observations.

In most previous identification studies of large samples of radio/X-ray sources, the spectral classification was generally rather coarse, given generally low spectral resolution and/or signal-to-noise ratio $(\mathrm{S} / \mathrm{N})$ acquired, and/or poor spectrophotometry calibration. For instance, weak or relatively narrow broadline components were hard to detect, leading to incorrect classifications between type I and type II AGNs. Moreover, emission line objects without an apparent broad line component were often classified collectively as narrow line radio galaxies (NLRGs), which are commonly thought to be the radio-loud counterparts of Seyfert II galaxies (e.g. Osterbrock 1976, Koski 1978, Urry \& Padovani 1995). However, this practice is vague and sometimes misleading, since it has been known for a long time that some radio galaxies have optical spectra that hardly qualify as NLRGs (or Seyfert II galaxies). Even from the early work on identification of radio sources, some radio galaxies were found to show spectra dominated by low-ionization lines, unlike Seyfert II galaxies (e.g. Costero \& Osterbrock 1977, Laing et al. 1994), especially in FR I sources (e.g. Hine \& Longair 1979, Laing et al. 1994). On the other hand, there also exists a distinct group of radio sources with weak [O III] line strength, termed as weak line radio galaxies (WLRG, Tadhunter et al. 1998). In fact, most of these objects, when observed with a better spectral resolution and $\mathrm{S} / \mathrm{N}$, turned out to be LINERs (Lewis et al. 2003). Rigorous classification of emission line galaxies should rely on the emission line ratio diagnostics (e.g. Baldwin et al. 1981; Veilleux \& Osterbrock 1987). This, however, requires good spectrophotometric calibration and sufficient spectral resolution and S/N, which could not be reached in some of the identification work of radio and X-ray sources in the past.

Furthermore, the ground-based slit or fiber spectra of moderately or under- luminous AGNs are often contaminated by starlight of the host galaxies. The spectra of host galaxy starlight could severely affect the detection and measurement of emission lines, by suppressing some weak emission lines (such as $H \beta$ ), distorting the line fluxes, and even generating spurious weak broad emission lines.

The Sloan Digital Sky Survey (SDSS, Stoughton et al.2002) has acquired optical spectra of about one million galaxies and quasars with homogeneous quality and reasonably good spectral resolution, as well as good spectrophotometric calibration accuracy ( $\sim 8 \%$, Vanden Berk et al. 2004). Furthermore, efficient algorithms have been developed to decompose nuclear emission line spectra from the host galaxy starlight, making it possible to accurately measure emission line fluxes and to recover weak emission line features. These improvements allow refined optical spectral classification of radio or X-ray sources. This is particularly relevant to low-luminosity AGNs (LLAGNs) with relatively weak emission lines. Identification of these sources with LLAGNs would complement the study of LLAGNs in general by providing useful radio and X-ray data.

In recent studies of X-ray-radio AGN samples, a few interesting new trends were found. One was the suggestion of the presence of flat-spectrum radio quasars (FSRQs) appearing to show spectral energy distributions (SEDs) typical of high-energy peaked BL Lac objects (HBLs) (e.g. Padovani et al. 2003, Perlman et al. 1998). Such objects were claimed to be mostly found in X-ray selected samples for their strong X-ray emission, and were missed in earlier pure radio-selected blazar samples. If the SEDs of these objects indeed similar to those of HBLs, they would present a challenge to the so-called "blazar sequence"a model in which the blazar SED is strongly correlated with the source power (Fossati et al.1998, Ghisellini et al. 1998). This is because objects with HBL-type SED at quasar luminosities are not a consequence of the model (Padovani et al. 2003). Independent confirmation of the presence of such FSRQs would still be worthwhile, although it is still a subject to actively debate whether the effective two-point spectral indices are good indicators of HBL-type SEDs (Maraschi et al.2008). Moreover, recently Yuan et al. (2008) found a fraction of very radio-loud narrow line Seyfert 1 galaxies (NLS1) in their small sample appearing to have HBL-type effective spectral indices (though at relatively low luminosities). A related question then is what is the fraction of NLS1 in FSRQs with "apparent" HBL-type SEDs. This can only be addressed by observations with sufficient $\mathrm{S} / \mathrm{N}$ ratios and spectral resolutions that resolve a relatively narrow broadcomponent $\left(<2000 \mathrm{~km} \mathrm{~s}^{-1}\right)$ in the presence of (strong) narrow Balmer lines. 
Motivated by the above issues, we carry out optical identification of unidentified sources in the RGB sample (Brinkmann et al.1997) using the SDSS spectroscopic data. The choice of the RGB sample is based on the following considerations. Firstly, the sample has refined VLA astrometry accuracy of $\pm 0.5^{\prime \prime}$ to ensure unambiguous matching with SDSS objects; also there is a large overlap of sky coverage of the RGB sample with the SDSS spectroscopic survey. Secondly, the RGB's high X-ray flux limit preferentially selects X-ray bright objects. This is essential for selecting FSRQs with "apparent” HBL-type spectral indices (Perlman et al. 1998; Padovani et al. 2003). Also, given its high thresholds in both the radio and X-ray bands, any identification with LLAGNs would place the objects in the regime at the higher-end of the luminosity function of LLAGNs, which are otherwise difficult to find owing to their rarity. We describe the RGB-SDSS sample and optical data analysis in Section 2, and present the results in Section 3. The results are summarized and discussed in Section 4, with emphases given to LLAGNs and FSRQs with HBL-type spectral indices. Through out the paper, we use a cosmology with $H_{0}=70 \mathrm{~km} \mathrm{~s}^{-1} M p c^{-1}, \Omega_{M}=0.3$, and $\Omega_{\Lambda}=0.7$.

\section{RGB-SDSS SAMPLE AND OPTICAL SPECTRAL ANALYSIS}

\subsection{RGB-SDSS sample}

The RGB catalog (Brinkmann et al. 1995) was constructed by cross-correlating the ROSAT All-Sky Survey (RASS) with the radio catalog created from the 1987 Green Bank (GB) survey maps (Gregory $\&$ Condon 1991, Gregory et al. 1996). The radio catalog consists of sources of $\geq 3 \sigma$ confidence with a flux limit ranging from $15 \mathrm{mJy}$ to $24 \mathrm{mJy}$ (at low declinations). The initial RGB sample is composed of 2127 matches with an angular separation $<100^{\prime \prime}$ between the X-ray and radio positions. Among them, 617 sources had been previously optically identified as extragalactic objects using NED by Brinkmann et al. (1995). The sample sources were further observed with the VLA to pin down their radio positions to an accuracy of $\pm 0.5^{\prime \prime}$ (Laurent-Muehleisen et al. 1997). Using the accurate VLA radio positions, a refined large sample of $1304 \mathrm{X}$-ray/radio sources from the RGB sample, mostly optically unidentified 1 , was presented in Brinkmann et al. (1997). This sample forms the parent sample of this study. We cross-correlated all the radio sources associated with the 1304 RGB X-ray sources in Brinkmann et al. (1997) with the SDSS DR5 spectroscopic databases of both galaxies and quasars. A matching criterion of $3^{\prime \prime}$ was used. This yielded 181 objects with SDSS spectra, 113 of which were assigned as QSOs, 49 as galaxies and 19 as unknown by the SDSS pipeline.

To estimate the radio spectral indice 2 of the objects, we also searched for their emission at $1.4 \mathrm{GHz}$ from the NVSS (NRAO VLA Sky Survey). A matching criterion of $3^{\prime}$ in radius was used when crosscorrelating the VLA and NVSS positions. Since the GB $5 \mathrm{GHz}$ survey had a resolution (beam size, $3.6^{\prime} \times 3.4^{\prime}$ ) larger than that of NVSS, for each match, we consider the following cases respectively to eliminate inconsistency in the estimation of source fluxes caused by the effect of different angular resolutions. First, if the NVSS counterpart is the only radio source within $3^{\prime}$ of the VLA position, the NVSS data is simply considered as its $1.4 \mathrm{GHz}$ flux. This is the case for the vast majority of sample objects (86). Second, we consider the case where there are more NVSS sources than matching NVSS counterparts at the VLA positions within $3^{\prime}$. If the summed flux of the former is significantly weaker than the latter, we used the total $1.4 \mathrm{GHz}$ radio flux within $3^{\prime}$ to calculate the spectral index; otherwise, we consider that the object's $5 \mathrm{GHz}$ flux is significantly contaminated by nearby sources and subsequently no spectral index can be inferred. As a result, we obtained the radio indices for 103 objects. Following the convention, flat- and steep-spectrum sources have $\alpha_{r} \leq 0.5$ and $\alpha_{r}>0.5$, respectively. The sample is summarized in Table 1, with some of the optical and radio data. The redshifts are measured from our own spectral data analysis (see below).

\footnotetext{
${ }^{1}$ In another identification attempt, Laurent-Muehleisen et al. (1998) spectroscopically observed and identified 169 objects with the McDonald $2.7 \mathrm{~m}$ and the Kitt Peak $2.1 \mathrm{~m}$ telescopes.

2 We assume a power law form $f_{\nu} \sim \nu^{-\alpha_{r}}$.
} 
Table 1 Source number of optical identification and classifications for the RGB-SDSS sample

\begin{tabular}{l|r}
\hline $\begin{array}{l}\text { classification } \\
(1)\end{array}$ & number \\
$(2)$ \\
\hline QSO(type I) & 84 \\
Seyfert I & 27 \\
NLSy1 & 2 \\
LINERs & 12 \\
Seyfert II & 2 \\
BL Lac & 32 \\
BL Lac candidate & 11 \\
Galaxy & 11 \\
\hline
\end{tabular}

\subsection{Optical spectral analysis}

SDSS spectra were taken through fibers with an aperture of $3^{\prime \prime}$ in diameter and have a wavelength coverage from 3800 to $9200 \AA$ and a resolution of $\mathrm{R} \sim 1800-2200$. For some objects, especially those assigned as galaxies by the SDSS pipe-line, their spectra include significant amounts of (or even are dominated by) starlight from the host galaxies. Thus, the removal of the stellar spectrum is essential for the proper detection and measurement of nuclear emission lines, especially when the lines, broad or narrow, are not strong. This is important for ensuring reliable spectral classification of identified objects. We consider objects as having non-negligible star-light contribution if their spectra show (possible) absorption lines of Ca K (3934 $)$ ), Ca K $+\mathrm{H} \epsilon(3970 \AA$ ), or the high-order Balmer line $\mathrm{H} \delta$ (with detection significance $>2 \sigma$ ). For proper modeling of host galaxy starlight, we used the algorithm developed at the University of Science of Technology of China, which was described in detail in Zhou et al. (2006) (see also Lu et al. 2006) and is summarized in Appendix A. The modeled host galaxy stellar spectrum is then subtracted from the SDSS spectrum.

For most of the RGB-SDSS objects, the SDSS spectra or the leftover spectra (modeled starlight spectrum subtracted) show significant emission lines. The emission line spectra are fitted using an updated version of the code as described in Dong et al. (2005), with improvements made for optimized recovery of weak emission lines. The method of the spectral fitting is also summarized in Appendix A. The Balmer emission lines are de-blended into a narrow and a broad component if the latter is present. If a broad line component is detected at the $\geq 5 \sigma$ confidence level, we regard it as genuine. As shown in Zhou et al. (2006), this analysis procedure yields reliable detection of emission lines and accurate measurements of the line parameters. The derived parameters of the common emission lines are given in Table 3 and Table 4 for the narrow- and broad-line components, respectively.

\section{RESULTS OF OPTICAL IDENTIFICATION AND CLASSIFICATION}

Thanks to the accurate astrometry of radio VLA positions and optical SDSS positions, the optical identifications of the RGB-SDSS objects are unambiguous. Here, we present detailed spectral classification of the 181 RGB-SDSS objects (see table 1). The sample is first separated into two groups: (i) emission-line objects with at least a few common significantly detected emission lines (e.g. $H \alpha, H \beta$, [O III], [N II], [S II]) detected significantly, with signal-to-noise ratio $\mathrm{S} / \mathrm{N}>3$ and equivalent width $E W \geq 5 \AA$ for at least one emission line (127 sources); and (ii) objects with weak or no emission lines (54 sources) (see below for the $E W$ threshold distinguishing Seyfert galaxies and BL Lac objects).

\subsection{Broad line AGNs}

Of the 113 emission line objects with a significant broad component (see table 4 ), there are 91 that have a broad component of the Balmer lines detected at $\mathrm{S} / \mathrm{N}>5$, and the others with $\mathrm{Mg}$ II broad emission lines. By broad component we mean that its line width is significantly broader than that of the corresponding narrow component of the line, typically several hundred kilo-meters per second. The smallest width of the broad component found in our sample is $1500 \mathrm{~km} \mathrm{~s}^{-1}$, still much broader than the narrow lines. We collectively classify these objects as broad line AGNs (BLAGNs), i.e. or type I AGN. They are further separated into 

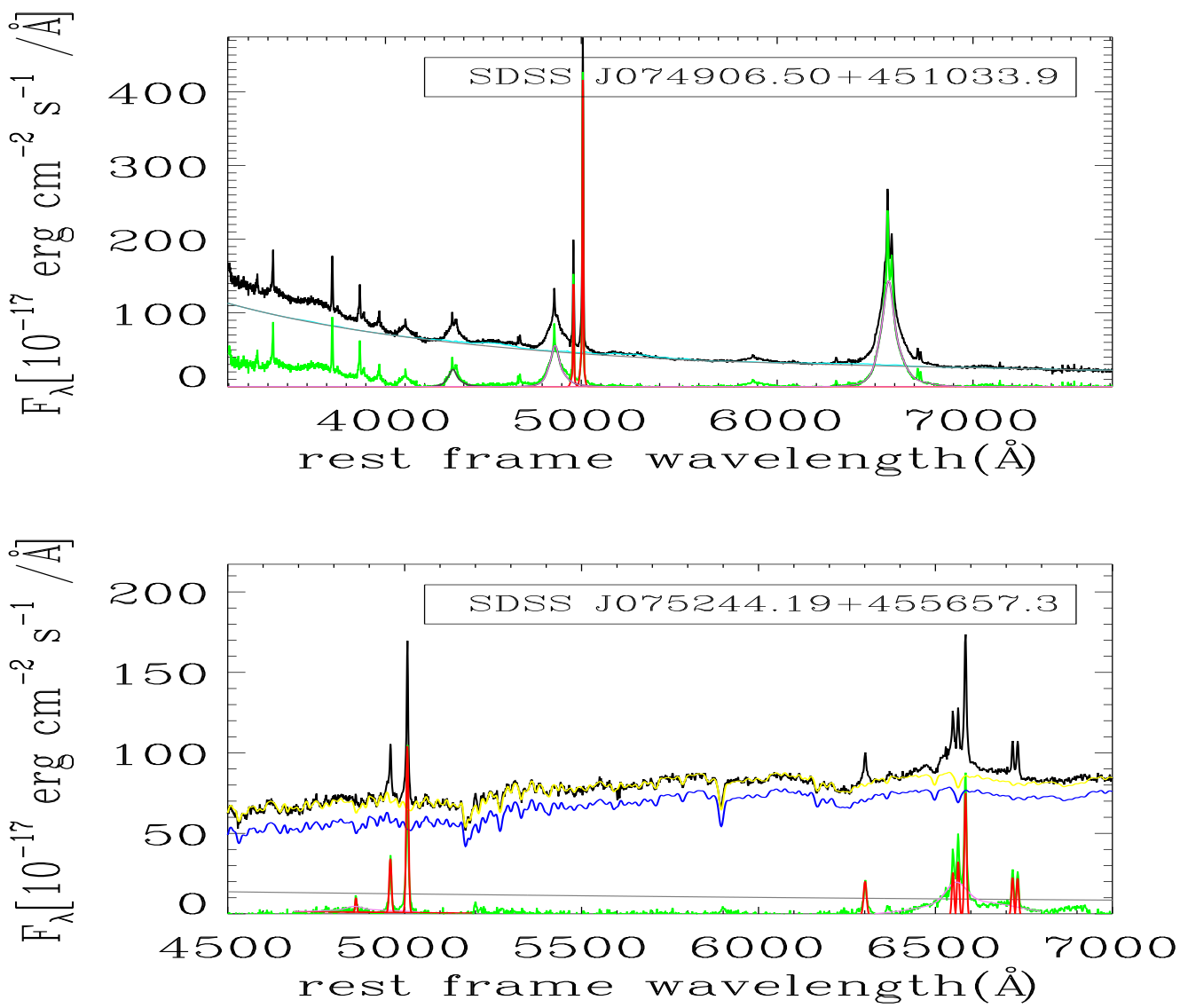

Fig. 1 Examples of the rest frame spectra (black) of identified broad line (type I) AGNs for a quasar (upper panel) and a BLRG (lower panel, with nuclear-starlight decomposition performed). Also plotted are the best-fit model components: starlight (blue), nuclear power-law continuum (grey) with the Fe II multiplets superimposed (cyan), the nuclear continuum plus starlight (yellow), residuals after subtraction of the nuclear continuum and starlight (green), broad (purple) and narrow (red) emission line components.

quasars and Seyfert I galaxies, using the common absolute magnitude dividing line $3 M_{\mathrm{B}}=-22.27$. Of the 84 objects qualifying quasar luminosity, all have radio-loudness $\mathrm{R}>10$ (defined as $\mathrm{R}=L_{5 G H z} / L_{4400 \AA}$, Kellermann et al. (1989) and thus are radio-loud. The remaining 29 are Seyfert I galaxies, i.e. broad line radio galaxies (BLRGs). As a demonstration, Fig.11 shows examples of the spectra of a quasar and a BLRG, respectively.

We note that among these BLAGNs two have the FWHM of $H \alpha$ or $H \beta$ smaller than $2000 \mathrm{~km} \mathrm{~s}^{-1}$ , namely, J094857.31+002225.5, J164442.53+261913.2. Their SDSS spectra reveal relatively strong Fe II multiplet and weak [O III] emission $([\mathrm{O} \mathrm{III}] / H \beta<3)$, satisfying the conventional criteria of NLS1s (Osterbrock \& Pogge 1985). It should be noted that NLS1 AGNs with strong radio emission are very rare, and were found to be present in small numbers only recently (see e.g. Komossa et al.2006, Whalen

\footnotetext{
${ }^{3}$ We used $M_{\mathrm{B}}=-21.5+5 \log \left(H_{0} / 100\right)$ (Peterson 1997). The B-band magnitude was transformed from the Galactic extinction corrected SDSS psf magnitude in the $\mathrm{u}$ and $\mathrm{g}$ band following $\mathrm{B}=\mathrm{g}+0.17(\mathrm{u}-\mathrm{g})+0.11$ (Jester 2005). The B-band absolute magnitude was calculated as $M_{B}=B-5 \log d_{L}-25-K(z)$, where $d_{L}$ is the luminosity distance and $K(z)$ is the K-correction, $K(z)=$ $2.5\left(\alpha_{\nu}-1\right) \log (1+z)$ (Peterson[1997)
} 
et al. 2006, Yuan et al. 2008). In fact, J094857.31+002225.5 and J164442.53+261913.2 were included in the radio-loud NLS1 sample of Yuan et al. (2008). Very recently, the gamma-ray emission from relativistic jets in J094857.31+002225.5 (PMN J0948+0022) has been detected by Fermi/LAT (Abdo et al. 2009).

\subsection{Narrow line radio galaxies}

For the remaining 14 emission-lined objects, no significant broad components of the lines can be detected. Traditionally, they were collectively referred to as NLRGs in previous works about their identification. Among them, nine have [O III] emission line equivalent widths smaller than $10 \AA$, and thus belong to the class of so-called weak-line radio galaxies (WLRGs) as suggested by Tadhunter et al. (1998). In fact, galactic nuclei with narrow emission line spectra are of heterogeneous types, including Seyfert II nuclei, low-ionization nuclear emission regions (LINERs, Heckman 1980), as well as star-forming galaxies. The nature of LINER is still a matter of debate, though recent studies tend to support the idea that the energy source is predominately from accretion on to a central black hole, as in normal AGNs (e.g. Ho et al. 1997). In this paper, we consider LINERs to be a subclass of AGNs, though they represent AGNs at a low level of activity.

Further classification of the narrow emission line nuclei of galaxies can be carried out based on a set of emission line ratios, as suggested by Baldwin et al. (1981) and developed by a number of authors (e.g. Veilleux \& Osterbrock 1987, Kewley et al.2001, Kauffmann et al.2003, Kewley et al.2006). Here, we adopt the most recent variant of this classification scheme as proposed by Kewley et al. (2006) and Kauffmann et al. (2003). In this scheme, AGNs (Seyferts and LINERs) are best separated from star-forming galaxies on the $[\mathrm{N} \mathrm{II}] / H \alpha$ versus $[\mathrm{O}$ III] $/ H \beta$ diagnostic diagram. Seyferts and LINERs are further distinguished using the $[\mathrm{O} \mathrm{I}] / H \alpha$ and $[\mathrm{S} \mathrm{II}] / H \alpha$ ratios, as shown in Fig.2] We found two Seyfert II and 12 typical LINER spectra(see Fig. 3 for example spectra). No star-forming galaxy is found, as expected for sources with strong $\mathrm{X}$-ray and radio emission as studied here. All of the nine WLRGs are classified as LINERs. Moreover, we also examined the LINER classification using the traditional criteria of [O II] $\lambda 3727 /[\mathrm{O}$ III] $\lambda 5007 \geq 1$ and [O I] $\lambda 6300 /[\mathrm{O}$ III] $\lambda 5007 \geq 1 / 3$ of Heckman (1980), and found that these criteria were also met for most of the LINERs. It should be noted that a few of the LINERs identified here show somewhat higher ionization atypical of LINERs. In fact, these objects are located very close to the border line separating LINERs and Seyferts in the diagnostic diagram of Kewley et al. (2006) and Kauffmann et al. (2003) and may have been classified as Seyferts if other classification criteria were used, e.g. Veilleux \& Osterbrock (1987), Heckman (1980) and Ho et al. (1997). Thus they are actually border-line objects between Seyferts and LINERs. We further point out that the LINER J151838.90+404500.2 was classified as Seyfert I galaxy by Laurent-Muehleisen et al. (1998); however, no significant broad component is found in its SDSS spectra.

We notice that the host galaxies of some sources identified with LINERs are associated with clusters of galaxies, as given by NED (Crawford et al. 1999, Koester et al. 2007); these are J111421.76+582319.8, J111908.94+090022.8, J113518.79+125311.1, J132419.67+041907.0, $\mathrm{J} 153253.78+302059.3, \mathrm{~J} 160239.61+264606.0$ and J172010.03+263732.0. Their host galaxies are most likely giant ellipticals in the centers of the clusters. For these objects the X-ray fluxes are mostly the diffuse emission of the clusters. Three of the objects, namely, J111421.76+582319.8, J153253.78+302059.3 and J172010.03+263732.0, were previously observed in optical by Crawford et al. (1999), and the SDSS spectra and the narrow line ratios are consistent with those presented in Crawford et al. (1999). Of particular interest, J111421.76+582319.8 is in a merging system, with two nuclei separated by $2.3^{\prime \prime}$ at nearly the same redshift. Its SDSS image is shown in Fig.4 Three of the classified LINERs (J113518.79+125311.1, J150324.77+475829.6 and J085004.65+403607.7) have the CaII H \& K break values smaller than 0.40, indicating possible contribution of a non-thermal (BL Lac) component in their optical emission, as previously found in J150324.77+475829.6 (Edge et al.2003).

\subsection{BL Lac objects and normal galaxies}

The second group of objects, those without significant emission lines, broadly show two kinds of spectra: a nearly featureless continuum (see table 7 and see Fig.5 for example spectra) and a stellar spectrum with absorption lines. In the case where a prominent featureless continuum (unlike stellar spectrum) is present, 

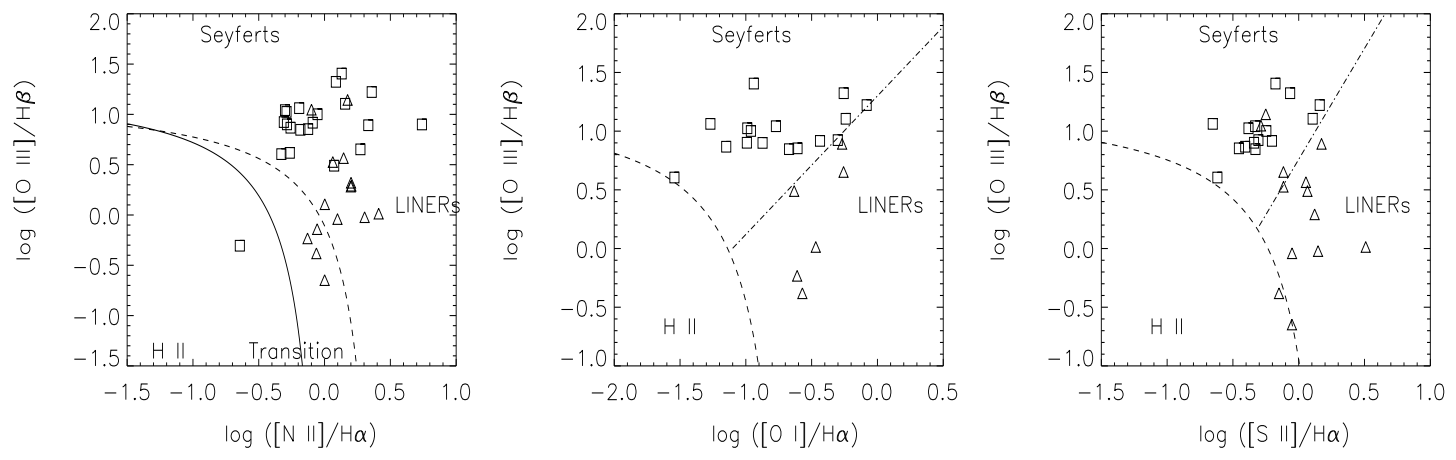

Fig. 2 Diagnostic diagrams of emission line ratios separating Seyfert galaxies, LINERs, and star-forming galaxies for emission line objects in the RGB-SDSS sample. The dividing schemes are adopted from Kauffmann et al. (2003) and Kewley et al. (2006). The solid line separates star-forming galaxies from Seyferts and LINERs (Kauffmann et al.2003), while the dashed line represents the maximum star-formation given by Kewley et al. (2001). The dashed-dotted line separates Seyferts from LINERs (Kewley et al. 2006). Plot symbols: squares for BLRG and triangles for NLRG.

we attribute it to non-thermal emission from BL Lac objects (see Fig.6for example spectra). In case of the absence of an apparent featureless continuum, we test its possible presence against the starlight using the contrast of the CaII H \& K absorption lines, i.e. the CaII H \& K break (Marcha et al.1996, Landt et al.2002, Collinge et al.2005). The CaII H \& K break is defined as

$$
\begin{gathered}
C_{\nu}=0.14+0.86 C_{\lambda} \\
C_{\lambda}=\left(f_{\lambda,+}-f_{\lambda,-}\right) / f_{\lambda,+}
\end{gathered}
$$

where $f_{\lambda,-}$ and $f_{\lambda,+}$ are the average flux densities in the rest-frame wavelength regions 3750-3950 and 4050-4250 $\AA$. This method is justified because the CaII H \& K break of elliptical galaxies should always be grater than 0.4 (Dressler \& Shectman 1987). The inclusion of starlight in the spectrum also dilutes the emission lines of AGNs. We adopt the method suggested by Marcha et al. (1996) to further distinguish BL Lac objects and Seyfert galaxies by considering both the CaII H \& K break and the equivalent width of emission lines, if any exist. The criteria are illustrated in Fig.7 as from Marcha et al. (1996), which shows the CaII $\mathrm{H} \& \mathrm{~K}$ break versus the equivalent width of the strongest line. Using this criteria, we classify objects with a CaII H \& K break greater than $40 \%$ (without a significant non-thermal continuum) as 'normal' galaxies. Those with the CaII $\mathrm{H} \& \mathrm{~K}$ break $<25 \%$ and the rest frame emission line $E W<5 \AA$ are classified as BL Lac objects; and those with the break between $25 \%$ and $40 \%$ and $E W<5 \AA$ are classified as BL Lac object candidates. Those with line $E W$ s marginally exceeding $5 \AA$ are classified, depending the CaII H \& K break, as either BL Lac objects candidates (smaller line EW) or LINERs or Seyfert galaxies (larger line EW) (see fig77). As a result, we found 32 BL Lac objects, 11 BL Lac candidates, and 11 'optical normal' galaxies.

\section{SUMMARY AND DISCUSSION}

\subsection{Summary of optical identification}

The identification of the RGB-SDSS sample objects is summarized in Table2. For 74 sources, the identifications are presented for the first time. There are 84 radio-loud quasars (45 FSRQs), 27 BLRGs, 2 radio-loud NLS1 galaxies, 14 NLRGs comprising 12 LINERs and 2 Seyfert II galaxies), 32 BL Lac objects and 11 BL Lac candidates, 11 'optical normal' galaxies (at least 6 in clusters). Thus the vast majority of the RGB 

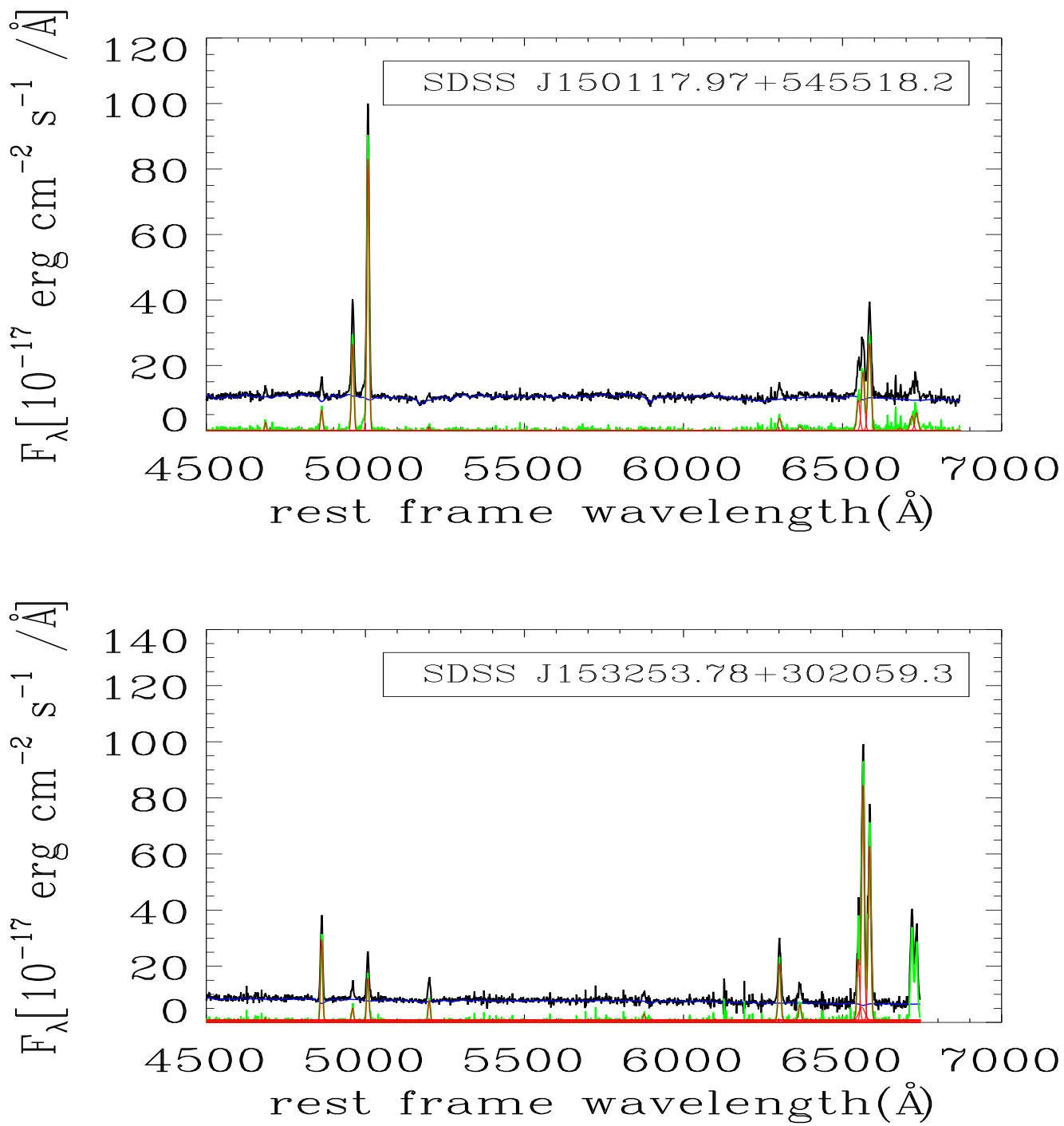

Fig. 3 Example spectra of identified narrow line AGNs in the rest frame for a Seyfert II galaxy (upper panel) and a LINER (lower panel). See Fig.1 for the plot coding.

sources are identified with radio-loud AGNs, particularly blazars, confirming previous results. The advantage of our result is the refined spectroscopic classification based on emission line ratios for NLRGs. Most of these NLRGs have weak emission lines (WLRGs) and have typical optical LINER spectra(12/14).

Of the RGB-SDSS sample, 36 sources have been previously identified by Laurent-Muehleisen et al. (1998) using 2-m class telescopes. Our results are in good agreement with theirs except for two objects (see Table 6). Furthermore, in the course of our work, Turriziani et al. (2007) published results of the identification of the ROXA sample, which was compiled from the RASS and the NVSS radio survey, and also made use of SDSS spectroscopic data. We note that 80 objects in that sample are included in our RGB-SDSS sample. For most of them (69 source), our classifications are consistent with those of Turriziani et al. (2007), while this is not the case for the remaining sources (11), as listed in Table6. It 


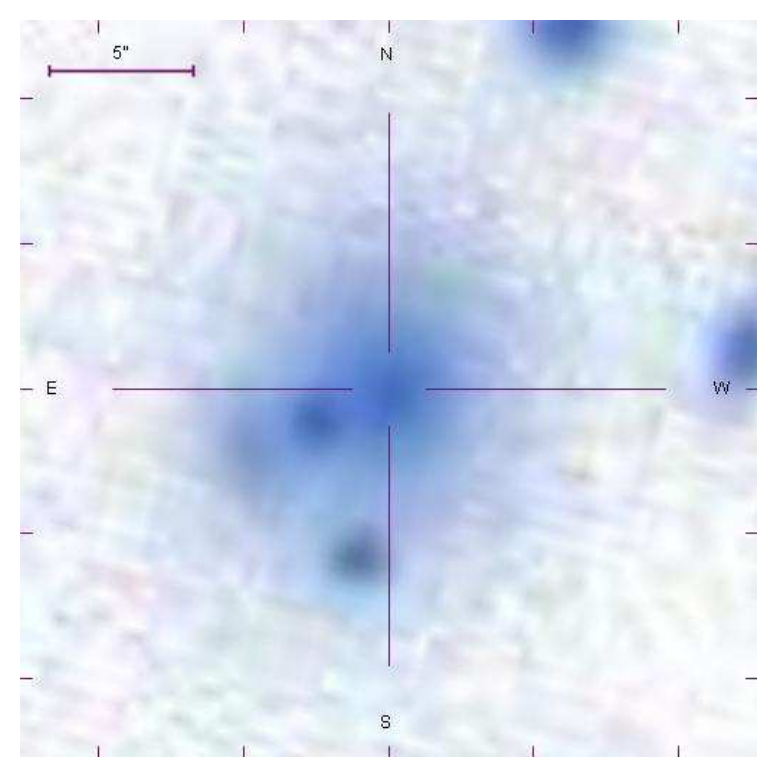

Fig. 4 Optical SDSS image of the LINER SDSS J111421.76+582319.8 (marked by the cross) shows that it is in a merging system with one or more other galaxies/cores.

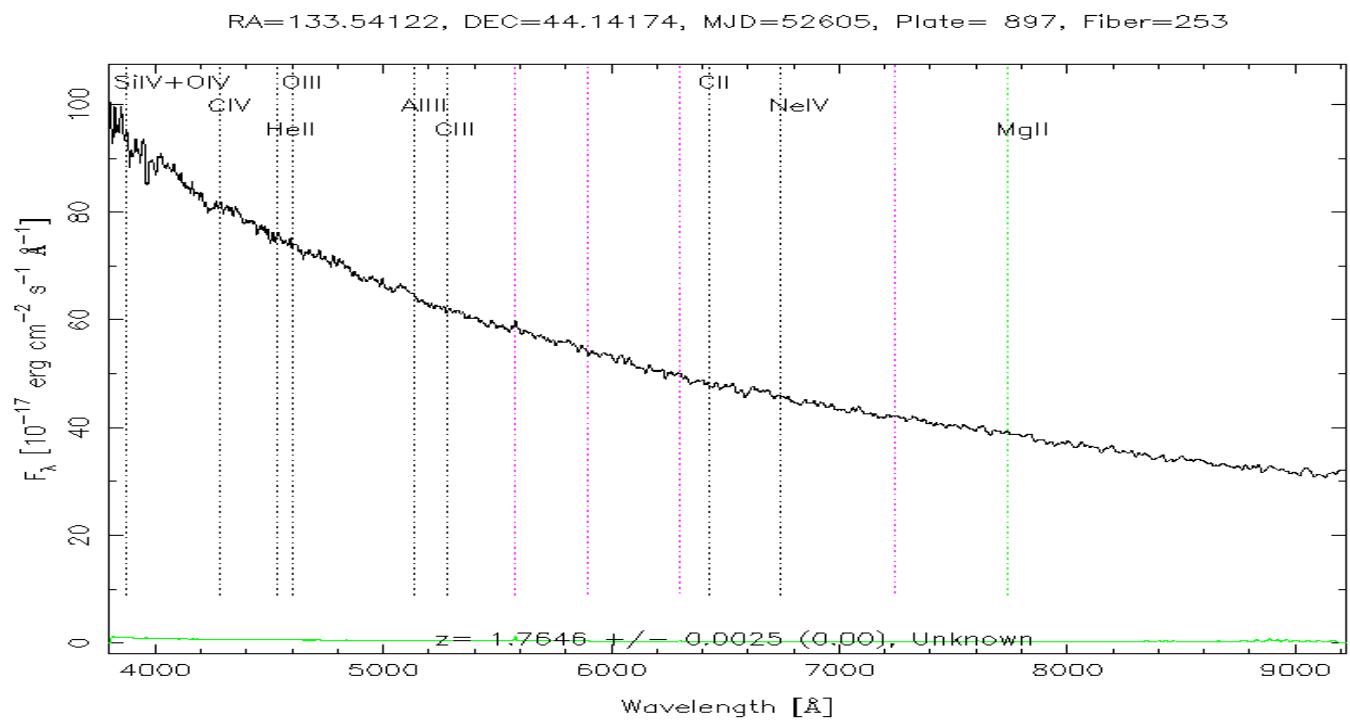

Fig. 5 Example SDSS spectrum of a BL Lac object with a featureless continuum.

should be pointed out that Turriziani et al. (2007) simply adopted the classification and spectral parameters given by the SDSS pipeline, which are coarse in general and even incorrect in some cases. We thus argue that our results are more accurate and reliable, especially for AGNs with weak emission lines or with narrower broad-lines than usual. 

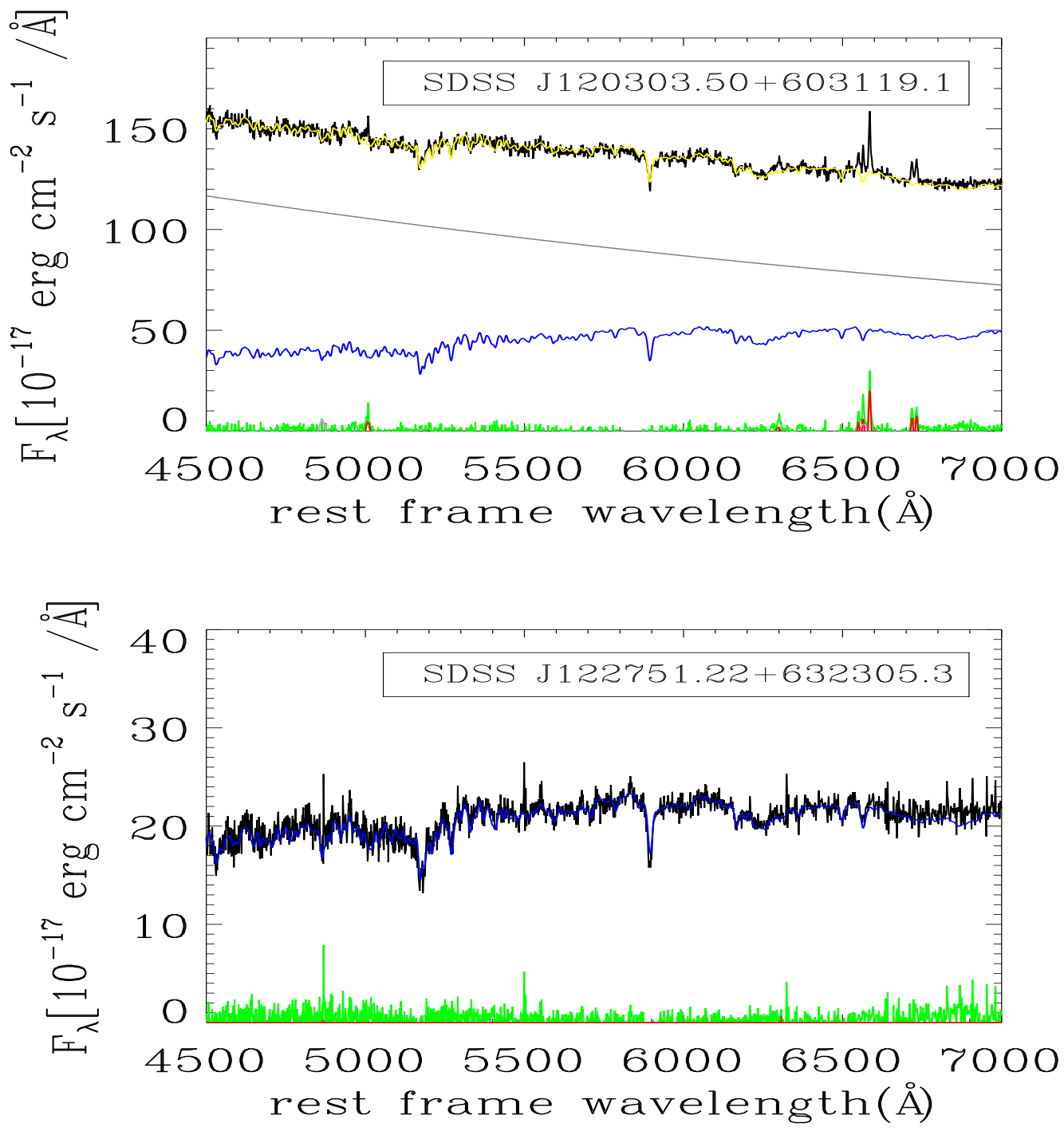

Fig. 6 Example spectra of an identified weak-lined BL Lac object (upper panel) and a normal galaxy (lower panel) in the rest frame. See Fig.1 for plot coding.

\subsection{Broad band SED}

From the radio, optical, and X-ray data of the sample objects, we calculated their broadband effective spectral indices $\alpha_{o x}, \alpha_{r o}$ and $\alpha_{r x}$ between $5 \mathrm{GHz}$ radio, $5000 \AA$ optical, and $1 \mathrm{keV} \mathrm{X-ray} \mathrm{using} \mathrm{the} \mathrm{monochro-}$ matic luminosities,

$$
\begin{gathered}
\alpha_{o x}=-\frac{\log \left(L_{1 \mathrm{keV}} / L_{5000 \AA}\right)}{\log \left(\nu_{1 \mathrm{keV}} / \nu_{5000 \AA}\right)}=-0.384 \log \left(\frac{L_{1 \mathrm{keV}}}{L_{5000 \AA}}\right) \\
\alpha_{r o}=-\frac{\log \left(L_{5000 \AA} / L_{5 G H z}\right)}{\log \left(\nu_{5000 \AA} / \nu_{5 G H z}\right)}=-0.196 \log \left(\frac{L_{5000 \AA}}{L_{5 G H z}}\right)
\end{gathered}
$$




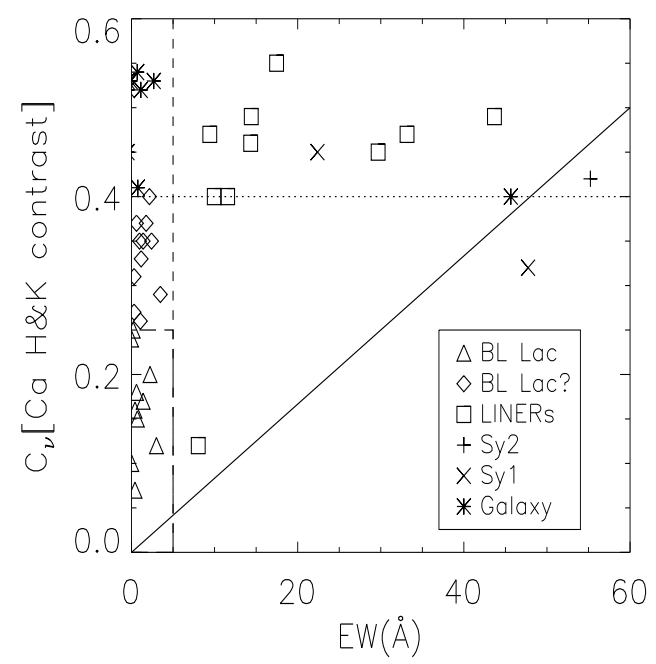

Fig. 7 The Ca H\&K break contrast versus the EW of the strongest emission line for objects without significant emission lines in the RGB-SDSS sample. The EW distribution is only shown out to EW $=60 \AA$ as in Marcha et al. (1996). The solid line represents the expected decrease for the EW of an emission line in the presence of an increasing amount of continuum following Marcha et al. (1996). The dotted line marks the Ca H\&K break contrast $C_{\nu}=0.40$ and the short dashed line marks EW $=5 \AA$. The area enclosed by the long dashed line corresponds to EW $\leq$ $5 \AA$ and Ca H\&K break contrast $\leq 0.25$, as used by Stocke et al. (1991) for the classification of BL Lac. Plot symbols: triangles for BL Lacs, diamonds for BL Lac candidates, squares for LINERs, plus signs for Sy IIs, crosses for Sy Is, and asterisks for galaxies.

$$
\alpha_{r x}=-\frac{\log \left(L_{1 \mathrm{keV}} / L_{5 \mathrm{GHz}}\right)}{\log \left(\nu_{1 \mathrm{keV}} / \nu_{5 \mathrm{GHz}}\right)}=-0.130 \log \left(\frac{L_{1 \mathrm{keV}}}{L_{5 \mathrm{GHz}}}\right)
$$

The choice of frequencies, as introduced in Ledden \& Odell (1985), makes it easy to compare with the results of Padovani et al. (2003). The optical monochromatic fluxes at $5000 \AA$ in the rest frame were derived from the Galactic extinction corrected SDSS PSF magnitude in the i-band using the extinction map of Schlegel et al. (1998), assuming an optical spectral index $\alpha_{\mathrm{o}}=0.5$ for the K-correction in the frequency domain. The monochromatic X-ray fluxes were derived from the integrated $0.1-2.4 \mathrm{keV}$ fluxes corrected for Galactic absorption which are given in Brinkmann et al. (1997); for this, the X-ray spectral indices were also taken from Brinkmann et al. (1997) if available (estimated from the hardness ratios) or assumed to be $\alpha_{\mathrm{x}}=1.2$ otherwise. For the radio spectral indices, the estimated $1.4-5 \mathrm{GHz}$ indices were used if available; otherwise a slope of 0.5 was assumed.

Figure 8 shows $\alpha_{\text {ro }}$ versus $\alpha_{\text {ox }}$ for the FSRQs and SSRQs, as well as BL Lac objects identified in this work. It can be seen that the distributions of the AGNs in the RGB-SDSS sample are very consistent with those found in previous studies (e.g. Brinkmann et al. 1995, Padovani et al. 2003) on the $\alpha_{\text {ro }}-\alpha_{\text {ox }}$ plane. The nominal dividing line between LBL (low-energy peaked BL Lac objects) and HBL (dashed $\alpha_{\mathrm{rx}}=0.78$ ) are indicated (solid lines), as well as the more strict locus for HBL, following Padovani et al. (2003). The figure shows that while many of the blazars (FSRQs and BL Lac objects) follow the trend of LBL, there are indeed a number of objects falling within the locus of HBL, including some FSRQs. Therefore, our result is consistent with that of Padovani et al. (2003) in the presence of FSRQs with the broad band two-point spectral indices resembling those of HBL.

One would like to know if these unusual FSRQs differ from normal FSRQs in terms of any other properties. We examined the distributions of the central black hole mass $\left(M_{\mathrm{BH}}\right)$ and the luminosity of the 
$\operatorname{BLR}\left(L_{\mathrm{BLR}}\right)$. The $M_{\mathrm{BH}}$ are estimated using the width and luminosity of the broad $\mathrm{H} \alpha$ or $\mathrm{H} \beta$ line, following Vestergaard \& Peterson (2006); Greene \& Ho (2007); and McGill et al. (2008), for FSRQs with measured $\mathrm{H} \alpha$ or $\mathrm{H} \beta$ lines. The $L_{\mathrm{BLR}}$ were calculated following the method suggested by Celotti et al. (1997) 4 . The distributions of $M_{\mathrm{BH}}$ and $L_{\mathrm{BLR}}$ for both the HBL-like FSRQs and the classical FSRQs are shown in Fig.9 and Fig.10 respectively. As can be seen, and also confirmed by the K-S (Kolmogorov-Smirnov) test, the $M_{\mathrm{BH}}$ and $L_{\mathrm{BLR}}$ distributions are indistinguishable for these two groups of FSRQs. We also compared the distributions of the Eddington ratios $5 L_{b o l} / L_{e d d}$ for the HBL-like FSRQs (median 0.105) and classical FSRQs (median 0.029) and concluded that they are marginally indistinguishable (with a probability level of 0.03 by the K-S test).

In light of the finding of Yuan et al. (2008) that a fraction of the very radio-loud NLS1 AGNs in their sample has an HBL-like $\alpha_{\mathrm{ro}}-\alpha_{\mathrm{ox}}$ distribution, it would be interesting to ask whether there is a link between NLS1s (or the narrowness of broad emission lines) and FSRQ with HBL-like SED. Figure11 shows the line-width distribution of the broad lines $(\mathrm{H} \alpha, \mathrm{H} \beta)$ for the normal FSRQs including Seyferts (dashed line) and the 14 candidate HBL-like FSRQs (solid line). The median line width of the HBL-like FSRQs is $3699 \mathrm{~km} \mathrm{~s}^{-1}$, smaller than $6159 \mathrm{~km} \mathrm{~s}^{-1}$ for normal FSRQs and Seyferts. However, the sample size is too small to confirm the difference; the K-S test gives a probability level of 0.34 . Thus, our sample is too small to give a conclusive answer to this question.

However, it should be pointed out that whether or not the broad band spectral indices are good indicators of HBL-type blazars is still a matter of active debate (for a different view, see Maraschi et al. (2008)). Therefore, the determination of whether these objects are genuine HBL-type FSRQs has to await proper estimation of the synchrotron peak frequency by obtaining the broad band SED data in the future.

\subsection{LINERs in the RGB-SDSS sample}

In previous identification work performed by Laurent-Muehleisen et al. (1998) and also by some others, objects without broad permitted emission lines were generally classified as NLRGs. Here, we are able to break this degeneracy and further classify them into Seyfert II nuclei and LINERs, based on their emission line ratios. Of particular interest, our results show that the majority of the narrow emission-line galaxies are identified with LINERs, and only a small fraction are identified with Seyfert II nuclei. Furthermore, all of those with weak emission lines (WLRGs) are in fact LINERs. This result is consistent with that of Lewis et al. (2003), who found that a large fraction $(\sim 50 \%-75 \%)$ of WLRGs is in fact LINERs, whenever a high quality optical spectrum was obtained.

The nature of LINERs is not yet well understood, but recent studies tend to suggest that a significant fraction of them consists of low-luminosity AGNs (LLAGNs), i.e. powered by black hole accretion (e.g. see Ho (2008) for a recent review). The radio properties of LINERs have been studied extensively over the years. It has been established that they often show radio core emission (e.g. Heckman 1980, Nagar et al.2005) or sometimes even sub-parsec-scale jets (e.g. Falcke et al.2000; Nagar et al.2005), mostly with flat or event inverted spectra (e.g. Nagar et al.2000, Falcke et al.2000). A detection rate of compact radio cores as high as $44 \%$ was found in LINERs, similar to that in Seyferts (Nagar et al.2005). Given that LINERS are radio emitters with flat spectra, the identification of some of the RGB sources with LINER is not surprising. However, for the majority of LINERs, the radio powers are generally quite modest—mostly in the range of

4 Following Celotti et al. (1997),

$$
L_{B L R}=\sum_{i} L_{i, o b s} \frac{<L_{B L R}^{*}>}{\sum_{i} L_{i, e x t}^{*}}
$$

where $\sum_{i} L_{i, o b s}$ is the sum of the measured luminosities of the observed broad lines, scaled by the ratio of the estimated total luminosities $L_{B L R}^{*}$ to the estimated luminosities of the observed broad lines. Both estimates were taken from the quasar template spectrum of Francis et al. (1991) and $H \alpha \lambda 6563$ from Gaskell et al. (1981). From this approach, $L_{B L R}=25.26 L_{H \beta}$ or $L_{B L R}=$ $16.35 L_{M g I I}$ or $L_{B L R}=7.22 L_{H \alpha}$ nosity.

5 The bolometric luminosities $L_{b o l}$ were calculated following the relationship of Richards et al. (2006) from the continuum lumi-

$$
L_{b o l}=9.26 L_{5100 \AA}
$$

In order to eliminate the possible contamination of a non-thermal emission component from relativistic jets in FSRQs, we estimated the continuum luminosity from the emission line luminosity following the relations in Greene \& Ho (2005). 


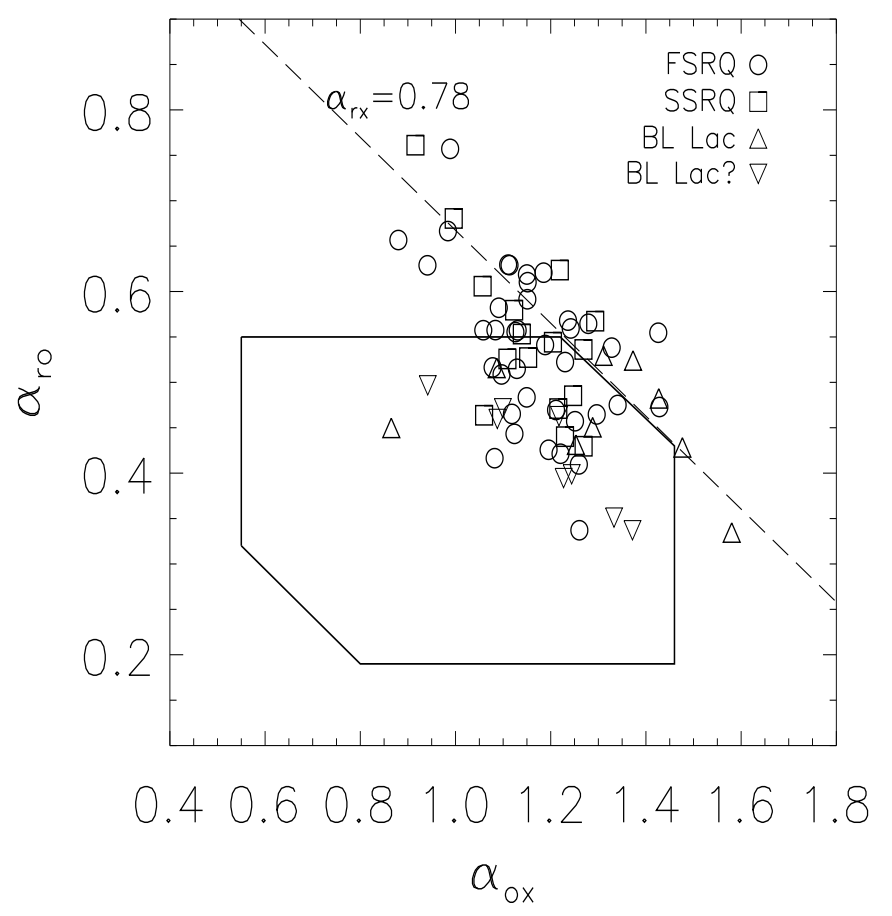

Fig. $8 \alpha_{\text {ro }}$ versus $\alpha_{\text {ox }}$ for the identified radio-loud quasars and BL Lac objects in the RGB-SDSS sample. The dashed line represents a constant $\alpha_{\mathrm{rx}}=0.78$, which is commonlyused to divide BL Lac objects into LBLs and HBLs.A more conservative, schematic locus for HBL is also indicated following Padovani et al. (2003) (solid line). Plot symbols: circles for FSRQs, squares for SSRQs, upside-down triangles for BL Lac candidates and triangles for BL Lac objects.

$10^{19-21} \mathrm{~W} \mathrm{~Hz}^{-1}$ at $5 \mathrm{GHz}$ for optically selected samples. The modest radio power is consistent with their low nuclear luminosities, in light of the suggested correlation between radio luminosity and emission line luminosity (e.g. Nagar et al.2005). In comparison, some of the LINERs identified in this work have very high radio core luminosities, $L_{5 \mathrm{GHz}}^{\text {core }}=10^{23}-10^{25} \mathrm{~W} \mathrm{~Hz}^{-1}$ at $5 \mathrm{GHz}$ (see Fig 12). They are of particular interest as they are possibly among the strongest radio emitters of the LINER population. In fact, these objects are at the higher-end of the radio luminosity function either of E/S0 galaxies (Sadler et al. 1989) or of LLAGNs (Nagar et al.2005), with both classes composed significantly of LINERs. We do not expect a significant relativistic beaming effect in these objects, since their radio core-dominance parameters are low or modest, as given in Laurent-Muehleisen et al. (1998). Thus, the large radio luminosities should be intrinsic.

It has been suggested that the radio power $L_{\mathrm{r}}$ of LINERs is correlated with the emission line luminosity (e.g. Nagar et al.2005). Indeed, the emission line luminosities (or the power of the NLR) of these LINERs are very high, $L_{\mathrm{H} \alpha+\mathrm{NII}}$ ranging from $10^{40.7}$ to $10^{42.8} \mathrm{erg} \mathrm{s}^{-1}$, on average $\sim 2$ orders of magnitude higher than those of the LINERs in the Palomar nearby galaxy sample of Ho et al. (1995) which were detected in radio by Nagar et al. (2005). We estimated their NLR luminosities following Celotti \& Fabian (1993) 6 , which have $\log L_{\mathrm{NLR}}\left(\mathrm{erg} \mathrm{s}^{-1}\right)=41.7-43.6$, with a median of 42.2. Interestingly, these values fall in between (with considerable overlaps) those of high-power FRI/FRII radio galaxies and normal low-power LLAGNs

${ }^{6} L_{N L R}=3\left(3 L_{[O I I] 3727}+1.5 L_{[O I I I] 5007}\right)$ 


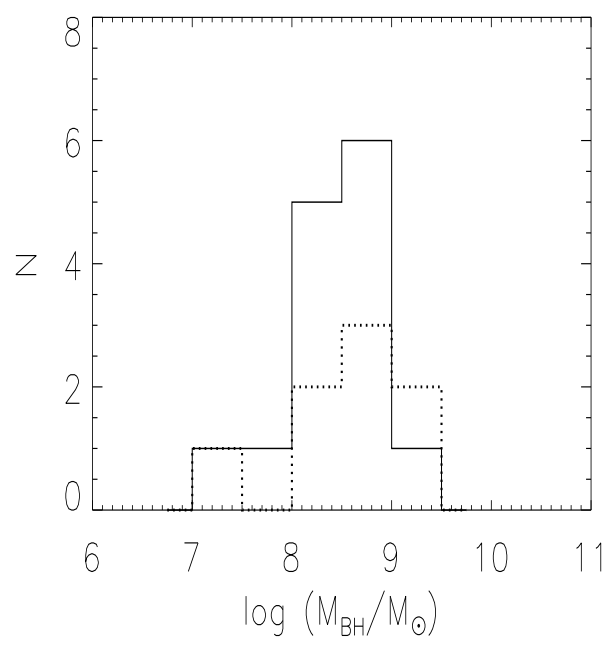

Fig. 9 Distribution of the black hole masses for normal FSRQs (dashed line) and those with the two-point spectral indices similar to that of HBL (solid line).

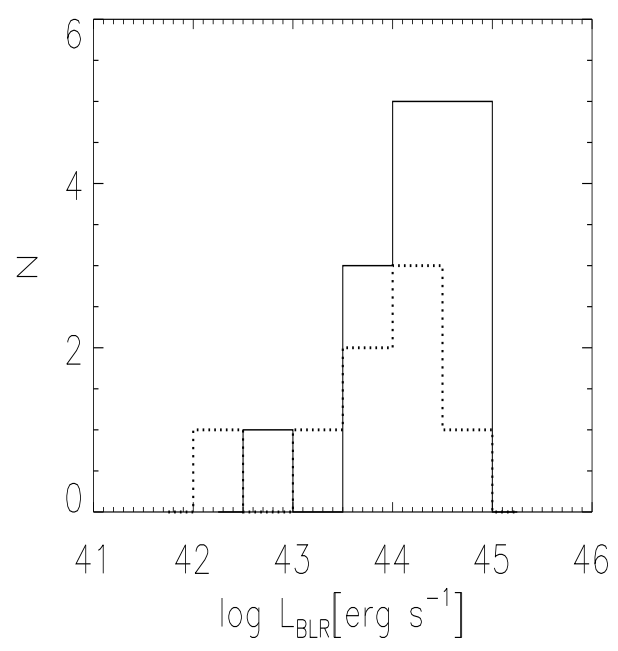

Fig. 10 Distribution of the luminosities of the broad line region for normal FSRQs (dashed line) and those with the two-point spectral indices similar to that of HBL (solid line)

as found in the Ho et al. (1995) sample and other optically selected LINER samples. Hence, they are the radio luminosities. This result is in line with the previous finding that LINERs and low-luminosity Seyferts follow the same radio-NLR-luminosity relation as FR I and FR II radio galaxies (e.g. Nagar et al. 2005), which is shown in Fig.13. It can be seen that our LINERs are located close to FR IIs, similar to the LINERs in non-elliptical host galaxies in the Palomar sample (see the left panel of their Fig. 3 in Nagar et al. (2005)). 


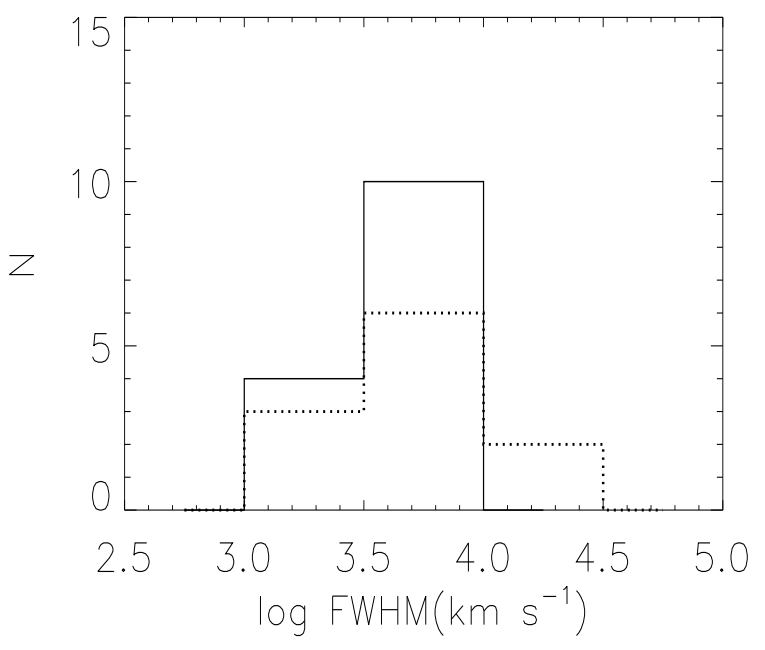

Fig. 11 Distribution of the broad line width (FWHM) for normal FSRQs (dashed line) and those with the two-point spectral indices similar to that of HBL (solid ).

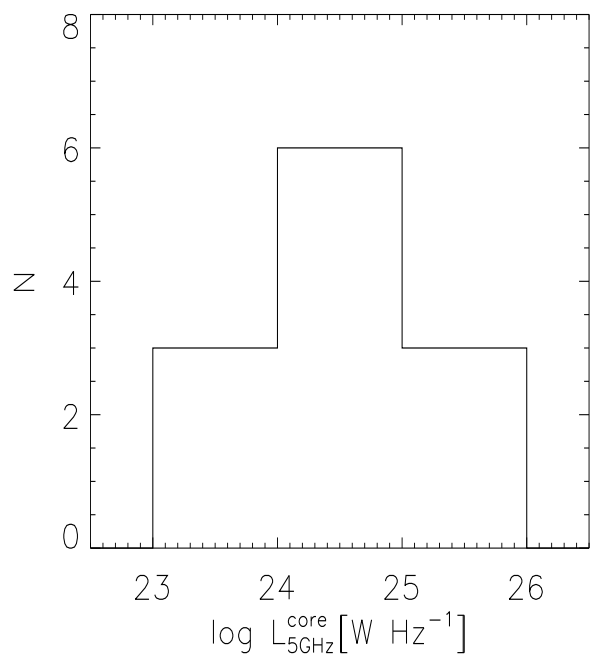

Fig. 12 Distribution of the radio core luminosities for the LINERs identified in this work.

A more fundamental relationship is the dependence of the radio power on both $M_{\mathrm{BH}}$ and the Eddington ratio, as suggested in e.g. Nagar et al. (2005). If this is the case, we anticipate that our LINERs must have the highest possible accretion rates for LINER and are harboring very massive central black holes. Among the 12 LINERs, 8 have reliable estimates of stellar velocity dispersion $\left(\sigma_{*}\right)$ from the central galactic stellar spectra within the $3^{\prime \prime}$ SDSS fibers, obtained via the nuclear-starlight decomposition algorithm (Appendix A). We estimated the central black hole mass using the well known $M_{\mathrm{BH}}-\sigma$ relation (Tremaine 


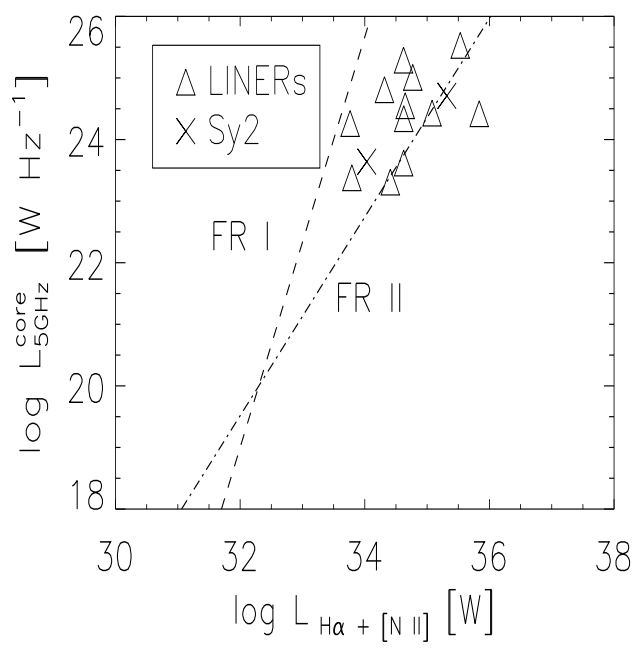

Fig. 13 Radio core luminosity versus narrow emission line $H \alpha+[N I I] \lambda \lambda 6548,6583$ luminosity for the LINERs (triangles) and Seyfert II galaxies (crosses) identified in this work. Also, plotted are the linear fits of the same relationship for FR I(dashed line) and FR II (dot-dashed line) radio galaxies from Zirbel \& Baum (1995); see also Nagar et al. (2005)

et al. 2002), which ranges from $\log \left(M_{\mathrm{BH}} / M_{\odot}\right)=8.4-9.7$ with a median of 9.3 , as listed in Table 5. Indeed, our LINERs are among those galaxies harboring the most massive central black holes, since this $M_{B H}$ range is in the high-end of the Black Hole mass function of galaxies at low redshifts $(\mathrm{z}<0.3)$ (McLure \& Dunlop 2004, Greene \& Ho 2007). Detailed investigation on the Eddington ratios, radio jet power, as well as other multi-wavelength properties is beyond the scope of this paper and will be pursued in future work.

The finding of radio luminous LINERs in this work is not surprising, given the relatively high cutoffs in the radio/X-ray selection of the RGB sample. We also compared our results with previous work in the literature. We calculated the radio luminosities of the WLRGs identified from the 2 Jy sample (Tadhunter et al.1998) which were further classified as LINERs by Lewis et al. (2003), and found that they have the same luminosity range as our LINERs. We therefore suggest that an effective way of finding powerful LINERs is to search for the associated LINERs with strong radio sources.

\section{ACKNOWLEDGMENTS}

This work is supported by the Chinese Natural Science Foundation through project No. NSF10533050, and by the National Basic Research Program of China - 973 Program 2009CB824800. Funding for the creation and the distribution of the SDSS Archive has been provided by the Alfred P. Sloan Foundation, the Participating Institutions, the National Aeronautics and Space Administration, the National Science Foundation, the U.S. Department of Energy, the Japanese Monbukagakusho, and the Max Planck Society. The SDSS is managed by the Astrophysical Research Consortium (ARC) for the Participating Institutions. The Participating Institutions are the University of Chicago, Fermilab, the Institute for Advanced Study, the Japan Participation Group, the Johns Hopkins University, Los Alamos National Laboratory, the Max-Planck-Institute for Astronomy (MPIA), the Max-Planck-Institute for Astrophysics (MPA), New Mexico State University, Princeton University, the United States Naval Observatory, and the University of Washington. This research has made use of the NASA/IPAC Extragalactic Database (NED) which is operated by the Jet Propulsion Laboratory, California Institute of Technology, under contract with the National Aeronautics and Space Administration. 
Table 2: RGB-SDSS Sample Objects and Identification

\begin{tabular}{|c|c|c|c|c|c|c|c|c|c|c|c|c|}
\hline $\begin{array}{c}\text { SDSS name } \\
\text { (1) }\end{array}$ & $\begin{array}{c}\mathrm{Z} \\
(2)\end{array}$ & $\begin{array}{c}\Delta_{r o} \\
(3)\end{array}$ & $\begin{array}{c}F_{6 \mathrm{~cm}} \\
(4)\end{array}$ & $\begin{array}{c}F_{20 \mathrm{~cm}} \\
(5)\end{array}$ & $\begin{array}{l}\alpha_{r} \\
(6)\end{array}$ & $\begin{array}{c}u \\
\text { (7) }\end{array}$ & $\begin{array}{c}g \\
(8)\end{array}$ & $\begin{array}{c}i \\
(9)\end{array}$ & $\begin{array}{l}M_{B} \\
(10)\end{array}$ & $\begin{array}{l}\lg R \\
(11)\end{array}$ & $\begin{array}{c}\text { SpecClass } \\
\text { (12) }\end{array}$ & $\begin{array}{l}\text { ref } \\
(13)\end{array}$ \\
\hline $\mathrm{J} 011354.50+132452.4$ & 0.6853 & 1.10 & $148 / 150$ & 247.8 & 0.40 & 18.67 & 18.38 & 18.52 & -23.70 & 3.09 & QSO & 2 \\
\hline J074906.50+451033.9 & 0.1921 & 0.17 & $50 /-$ & $\longrightarrow$ & 一 & 16.55 & 16.72 & 16.25 & -22.77 & 1.88 & QSO & 1 \\
\hline $\mathrm{J} 075244.19+455657.3$ & 0.0518 & 0.19 & $59 /-$ & 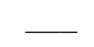 & - & 18.49 & 17.07 & 15.92 & -19.31 & 2.06 & Sy1 & \\
\hline J075448.86+303355.1 & 0.7956 & 0.12 & $108 /-$ & 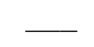 & — & 17.72 & 17.32 & 17.42 & -25.04 & 2.54 & QSO & 2 \\
\hline J081058.99+413402.7 & 0.5066 & 0.10 & $186 / 182$ & 219.7 & 0.15 & 18.56 & 18.42 & 18.37 & -23.07 & 3.18 & QSO & 2 \\
\hline $\mathrm{J} 081100.60+571412.5$ & 0.6110 & 0.15 & $305 / 375$ & 460.0 & 0.16 & 18.08 & 17.65 & 17.68 & -24.18 & 3.10 & QSO & \\
\hline J081432.11+560956.6 & 0.5093 & 0.11 & $47 / 43$ & 60.3 & 0.27 & 18.05 & 17.90 & 17.90 & -23.60 & 2.37 & QSO & 1 \\
\hline J081902.32+322637.2 & 0.6512 & 1.06 & $274 / 209$ & 194.4 & -0.06 & 21.36 & 20.75 & 19.72 & -21.17 & 4.30 & Sy1 & \\
\hline J081916.61+264203.2 & 0.5261 & 0.03 & $7 / 117$ & 254.6 & 0.63 & 18.93 & 18.61 & 18.66 & -22.93 & 1.83 & QSO & \\
\hline J085004.65+403607.7 & 0.2667 & 0.09 & $112 / 110$ & 139.3 & 0.19 & 20.98 & 19.75 & 18.22 & -20.21 & 3.45 & LINERs & \\
\hline J085036.20+345522.6 & 0.1450 & 0.27 & $28 / 20$ & 34.9 & 0.45 & 18.90 & 18.09 & 17.16 & -20.62 & 2.17 & BL Lac? & \\
\hline $\mathrm{J} 085039.95+543753.3$ & 0.3673 & 0.09 & $6 /-$ & - & - & 18.77 & 18.53 & 18.23 & -22.27 & 1.71 & Sy1 & \\
\hline J085348.18+065447.1 & 0.2232 & 0.12 & $220 /-$ & - & - & 20.30 & 19.75 & 18.41 & -19.94 & 3.74 & Sy1 & \\
\hline J085830.61+080422.8 & 0.4549 & 1.93 & $31 /-$ & & - & 18.77 & 18.45 & 18.28 & -22.79 & 2.40 & QSO & \\
\hline J090745.28+532421.4 & 0.7115 & 0.36 & $7 /-$ & - & 一 & 17.60 & 17.37 & 17.54 & -24.80 & 1.36 & QSO & \\
\hline J090835.85+415046.3 & 0.7337 & 0.04 & $114 / 222$ & 229.2 & 0.03 & 19.30 & 18.76 & 18.79 & -23.42 & 3.13 & QSO & 2 \\
\hline J090924.66+274402.8 & 0.3342 & 0.07 & $57 / 47$ & 117.6 & 0.74 & 18.42 & 18.50 & 18.42 & -22.16 & 2.67 & Sy1 & \\
\hline J090924.68+521632.6 & 0.4102 & 0.18 & $33 /-$ & - & - & 18.57 & 17.95 & 17.50 & -23.03 & 2.22 & QSO & 1,2 \\
\hline J091133.85+442250.1 & 0.2976 & 0.03 & $30 / 157$ & 446.4 & 0.84 & 18.76 & 18.72 & 18.24 & -21.67 & 2.47 & Sy1 & 2 \\
\hline J091925.65+110659.4 & 0.4247 & 0.09 & $31 / 31$ & 34.5 & 0.09 & 20.70 & 20.18 & 18.75 & -20.88 & 3.10 & BL Lac? & 2 \\
\hline
\end{tabular}




\begin{tabular}{|c|c|c|c|c|c|c|c|c|c|c|c|c|}
\hline $\begin{array}{c}\text { SDSS name } \\
\text { (1) }\end{array}$ & $\begin{array}{c}Z \\
(2)\end{array}$ & $\begin{array}{c}\Delta_{r o} \\
(3)\end{array}$ & $\begin{array}{c}F_{6 c m} \\
(4)\end{array}$ & $\begin{array}{c}F_{20 \mathrm{~cm}} \\
(5)\end{array}$ & $\begin{array}{l}\alpha_{r} \\
(6)\end{array}$ & $\begin{array}{c}u \\
(7)\end{array}$ & $\begin{array}{c}g \\
(8)\end{array}$ & $\begin{array}{c}i \\
(9)\end{array}$ & $\begin{array}{l}M_{B} \\
(10)\end{array}$ & $\begin{array}{l}\lg R \\
(11)\end{array}$ & $\begin{array}{c}\text { SpecClass } \\
\text { (12) }\end{array}$ & $\begin{array}{l}\text { ref } \\
(13)\end{array}$ \\
\hline J093430.68+030545.3 & 0.2252 & 0.07 & $83 / 119$ & 310.3 & 0.77 & 21.23 & 19.78 & 18.09 & -19.77 & 3.33 & LINERs & \\
\hline J093712.33+500852.1 & 0.2756 & 0.19 & $212 / 315$ & 170.8 & -0.49 & 19.65 & 19.10 & 18.07 & -21.04 & 3.47 & Sy 1 & 2 \\
\hline J095855.10+423704.0 & 0.6641 & 1.88 & $23 / 60$ & 98.0 & 0.39 & 19.09 & 18.77 & 18.93 & -23.25 & 2.43 & QSO & \\
\hline $\mathrm{J} 101244.30+422957.0$ & 0.3651 & 0.13 & $28 /-$ & - & - & 18.89 & 18.57 & 17.91 & -22.21 & 2.39 & BL Lac & 1,2 \\
\hline $\mathrm{J} 101258.34+393238.8$ & 0.1709 & 0.04 & $18 /-$ & - & - & 19.95 & 19.03 & 17.86 & -20.02 & 2.35 & BL Lac? & 2 \\
\hline $\mathrm{J} 102106.04+452331.8$ & 0.3642 & 0.61 & $22 / 82$ & 127.2 & 0.35 & 18.23 & 18.19 & 18.35 & -22.63 & 2.14 & QSO & 1,2 \\
\hline $\mathrm{J} 102235.57+454105.4$ & 0.7434 & 0.05 & $12 / 59$ & 145.6 & 0.73 & 21.06 & 20.81 & 19.96 & -21.44 & 2.98 & Sy 1 & \\
\hline $\mathrm{J} 102237.44+393150.1$ & 0.6036 & 0.90 & $40 /-$ & - & - & 17.68 & 17.32 & 17.01 & -24.49 & 2.09 & QSO & 2 \\
\hline $\mathrm{J} 102504.20+414332.6$ & 0.6818 & 0.04 & $6 / 20$ & 8.9 & -0.65 & 18.99 & 18.43 & 18.60 & -23.59 & 1.72 & QSO & 2 \\
\hline $\mathrm{J} 102523.04+040229.0$ & 0.2077 & 0.10 & $31 /-$ & 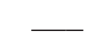 & - & 19.54 & 19.01 & 18.09 & -20.53 & 2.59 & BL Lac? & 2 \\
\hline $\mathrm{J} 103854.49+513937.4$ & 0.4701 & 0.07 & $21 / 22$ & 7.8 & -0.83 & 23.36 & 22.55 & 20.01 & -18.68 & 3.88 & Galaxy & \\
\hline $\mathrm{J} 104149.15+390119.5$ & 0.2084 & 0.10 & $23 / 19$ & 33.4 & 0.45 & 20.01 & 19.24 & 17.97 & -20.27 & 2.55 & BL Lac? & \\
\hline $\mathrm{J} 104334.81+343232.5$ & 0.7330 & 0.18 & $49 / 42$ & 113.5 & 0.80 & 20.92 & 20.18 & 20.23 & -21.96 & 3.33 & Sy 1 & \\
\hline $\mathrm{J} 104410.67+532220.5$ & 1.9033 & 0.10 & $307 / 437$ & 493.0 & 0.10 & 19.41 & 19.38 & 19.11 & -24.59 & 3.92 & QSO & 2 \\
\hline $\mathrm{J} 105431.89+385521.6$ & 1.3664 & 1.89 & $47 / 56$ & 61.2 & 0.07 & 17.24 & 17.15 & 17.17 & -26.26 & 2.17 & QSO & \\
\hline $\mathrm{J} 105837.73+562811.1$ & 0.1433 & 0.11 & $177 / 247$ & 228.0 & -0.06 & 16.84 & 16.42 & 15.95 & -22.34 & 2.30 & BL Lac & 1,2 \\
\hline $\mathrm{J} 110409.63+122157.5$ & 0.5908 & 0.15 & $33 /-$ & - & - & 17.78 & 17.30 & 17.48 & -24.45 & 1.99 & QSO & \\
\hline $\mathrm{J} 111421.76+582319.8$ & 0.2057 & 0.09 & $2 /-$ & 65.7 & 2.81 & 22.01 & 20.38 & 18.60 & -18.95 & 1.95 & LINERs & 3 \\
\hline $\mathrm{J} 111908.94+090022.8$ & 0.3316 & 0.30 & $13 /-$ & 22.3 & 0.43 & 23.13 & 21.09 & 19.09 & -19.19 & 3.07 & LINERs & 2 \\
\hline $\mathrm{J} 113251.05+631144.0$ & 0.1114 & 0.26 & $7 /-$ & - & - & 20.50 & 18.42 & 16.98 & -19.51 & 1.69 & Galaxy & \\
\hline $\mathrm{J} 113255.96+051539.6$ & 0.1009 & 0.06 & $78 /-$ & - & 一 & 20.04 & 18.35 & 17.10 & -19.43 & 2.70 & LINERs & \\
\hline
\end{tabular}




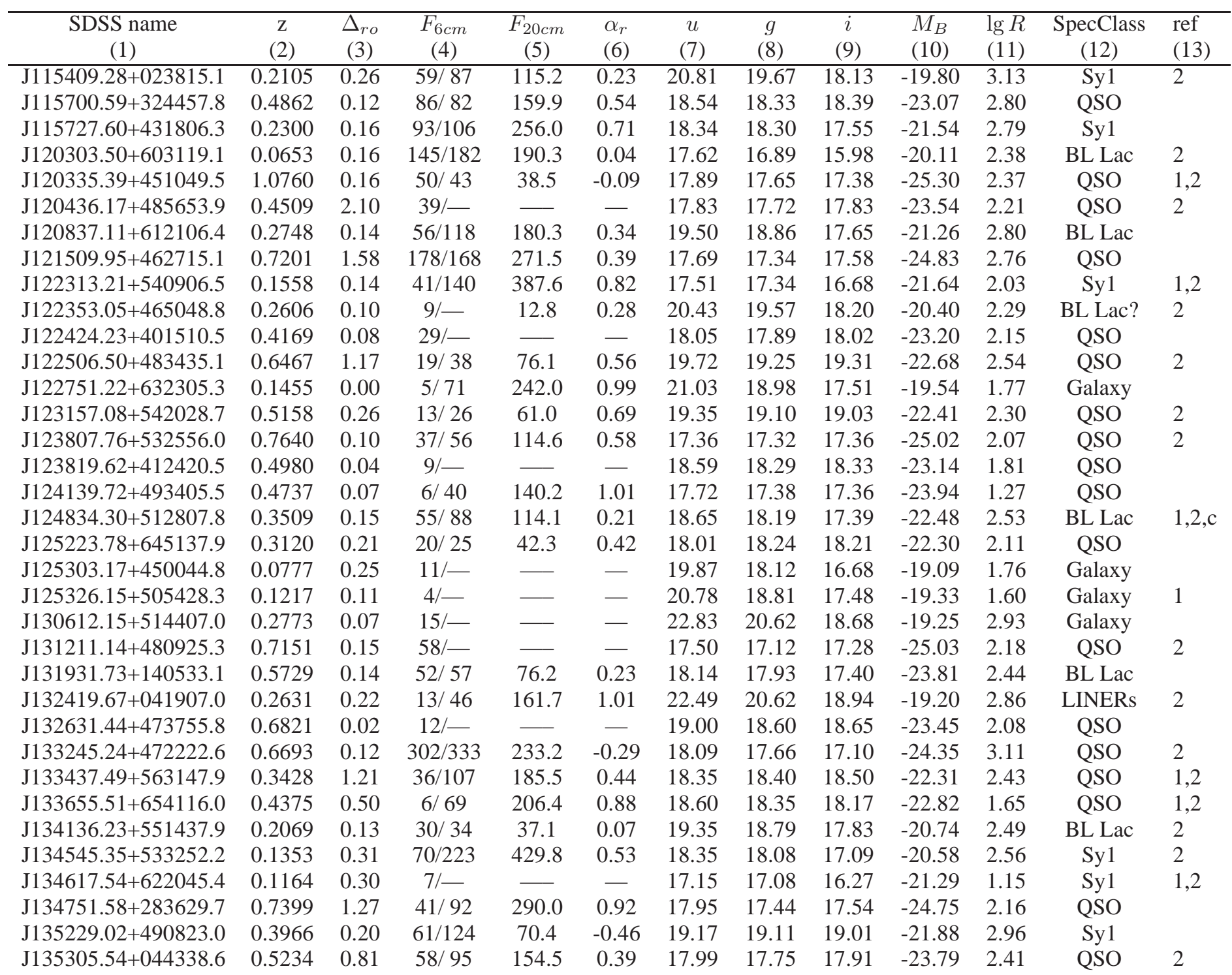




\begin{tabular}{|c|c|c|c|c|c|c|c|c|c|c|c|c|}
\hline $\begin{array}{l}\text { SDSS name } \\
\text { (1) }\end{array}$ & $\begin{array}{c}Z \\
(2)\end{array}$ & $\begin{array}{c}\Delta_{r o} \\
(3)\end{array}$ & $\begin{array}{c}F_{6 c m} \\
\text { (4) }\end{array}$ & $\begin{array}{c}F_{20 \mathrm{~cm}} \\
(5)\end{array}$ & $\begin{array}{l}\alpha_{r} \\
(6)\end{array}$ & $\begin{array}{c}u \\
(7)\end{array}$ & $\begin{array}{c}g \\
(8)\end{array}$ & $\begin{array}{c}i \\
(9)\end{array}$ & $\begin{array}{l}M_{B} \\
(10)\end{array}$ & $\begin{array}{l}\lg R \\
(11)\end{array}$ & $\begin{array}{l}\text { SpecClass } \\
\text { (12) }\end{array}$ & $\begin{array}{l}\text { ref } \\
(13)\end{array}$ \\
\hline $\mathrm{J} 135341.72+431052.5$ & 1.1140 & 0.16 & $15 /-$ & - & - & 17.44 & 17.24 & 17.10 & -25.79 & 1.69 & QSO & 1,2 \\
\hline $\mathrm{J} 141149.43+524900.1$ & 0.0765 & 0.25 & $96 / 354$ & 846.8 & 0.70 & 18.58 & 17.33 & 16.14 & -19.93 & 2.38 & Galaxy & 2 \\
\hline J141446.64+392818.6 & 0.6573 & 0.14 & $11 /-$ & - & - & 19.91 & 19.46 & 19.41 & -22.51 & 2.39 & QSO & 2 \\
\hline $\mathrm{J} 141628.66+124213.5$ & 0.3346 & 0.05 & $75 / 98$ & 110.2 & 0.09 & 17.41 & 17.44 & 17.65 & -23.21 & 2.37 & QSO & 2 \\
\hline $\mathrm{J} 141740.44+381821.1$ & 0.4495 & 0.06 & $173 / 95$ & 111.3 & 0.13 & 18.45 & 18.32 & 18.31 & -22.93 & 3.10 & QSO & 2 \\
\hline $\mathrm{J} 142314.19+505537.3$ & 0.2759 & 2.61 & $125 /-$ & - & - & 17.75 & 17.93 & 17.71 & -22.34 & 2.77 & QSO & 2 \\
\hline $\mathrm{J} 142421.17+370552.8$ & 0.2896 & 1.79 & $11 / 29$ & 78.6 & 0.80 & 20.41 & 19.65 & 18.20 & -20.56 & 2.41 & BL Lac? & 2 \\
\hline $\mathrm{J} 142606.19+402432.0$ & 0.6639 & 0.29 & $18 /-$ & + & - & 20.41 & 19.91 & 19.63 & -22.08 & 2.78 & Sy1 & \\
\hline $\mathrm{J} 142730.27+540923.7$ & 0.1060 & 0.80 & $23 / 26$ & 44.0 & 0.42 & 19.76 & 18.46 & 17.15 & -19.49 & 2.22 & Galaxy & 1 \\
\hline $\mathrm{J} 143726.14+504555.8$ & 0.7835 & 0.56 & $6 /-$ & - & - & 17.86 & 17.53 & 17.57 & -24.81 & 1.37 & QSO & 1 \\
\hline $\mathrm{J} 145958.43+333701.8$ & 0.6449 & 0.15 & $103 / 48$ & 15.3 & -0.92 & 16.88 & 16.62 & 16.77 & -25.35 & 2.22 & QSO & 1,2 \\
\hline $\mathrm{J} 150117.97+545518.2$ & 0.3386 & 0.15 & $18 /-$ & - & - & 20.56 & 19.92 & 18.15 & -20.64 & 2.74 & Sy2 & 2 \\
\hline $\mathrm{J} 150324.77+475829.6$ & 0.3445 & 0.08 & $9 / 56$ & 111.2 & 0.55 & 18.39 & 18.00 & 17.30 & -22.65 & 1.67 & LINERs & \\
\hline $\mathrm{J} 150455.56+564920.2$ & 0.3590 & 1.39 & $5 /-$ & - & - & 17.06 & 17.00 & 17.09 & -23.79 & 1.02 & QSO & 1 \\
\hline $\mathrm{J} 151017.82+422155.0$ & 0.4876 & 0.04 & $19 / 111$ & 262.5 & 0.69 & 21.60 & 21.20 & 19.85 & -20.17 & 3.30 & Sy 1 & 2 \\
\hline $\mathrm{J} 151830.93+483214.4$ & 0.5757 & 0.16 & $4 /-$ & & - & 18.66 & 18.27 & 18.32 & -23.44 & 1.46 & QSO & 1 \\
\hline $\mathrm{J} 151838.90+404500.2$ & 0.0652 & 0.07 & $25 /-$ & - & - & 19.28 & 17.63 & 16.56 & -19.21 & 1.91 & LINERs & 1 \\
\hline $\mathrm{J} 151844.76+461855.1$ & 0.8852 & 0.13 & $35 / 163$ & 436.0 & 0.79 & 20.20 & 19.91 & 19.80 & -22.67 & 3.10 & QSO & \\
\hline $\mathrm{J} 151913.35+362343.4$ & 0.2857 & 0.11 & $59 /-$ & - & 一 & 19.49 & 19.51 & 18.79 & -20.81 & 3.08 & Sy 1 & \\
\hline $\mathrm{J} 152556.22+591659.5$ & 0.9551 & 0.08 & $7 /-$ & - & - & 18.51 & 18.33 & 18.21 & -24.42 & 1.77 & QSO & \\
\hline $\mathrm{J} 153102.48+435637.6$ & 0.4520 & 0.10 & $18 /$ & - & - & 17.31 & 17.13 & 16.91 & -24.12 & 1.64 & QSO & 1,2 \\
\hline
\end{tabular}




\begin{tabular}{|c|c|c|c|c|c|c|c|c|c|c|c|c|}
\hline $\begin{array}{c}\text { SDSS name } \\
\text { (1) }\end{array}$ & $\begin{array}{c}\mathrm{Z} \\
(2)\end{array}$ & $\begin{array}{l}\Delta_{r o} \\
(3)\end{array}$ & $\begin{array}{c}F_{6 c m} \\
(4)\end{array}$ & $\begin{array}{c}F_{20 \mathrm{~cm}} \\
(5)\end{array}$ & $\begin{array}{l}\alpha_{r} \\
\text { (6) }\end{array}$ & $\begin{array}{c}u \\
(7)\end{array}$ & $\begin{array}{c}g \\
(8)\end{array}$ & $\begin{array}{c}i \\
(9)\end{array}$ & $\begin{array}{l}M_{B} \\
(10)\end{array}$ & $\begin{array}{l}\lg R \\
(11)\end{array}$ & $\begin{array}{c}\text { SpecClass } \\
\text { (12) }\end{array}$ & $\begin{array}{l}\text { ref } \\
\text { (13) }\end{array}$ \\
\hline $\mathrm{J} 160658.30+271705.5$ & 0.9335 & 0.17 & $143 / 228$ & 178.0 & -0.20 & 19.23 & 18.88 & 18.79 & -23.79 & 3.30 & QSO & \\
\hline $\mathrm{J} 160813.79+292126.3$ & 1.2006 & 1.08 & $12 /-$ & 38.9 & 0.95 & 18.87 & 19.08 & 18.67 & -24.16 & 2.33 & QSO & \\
\hline $\mathrm{J} 161706.32+410647.0$ & 0.2667 & 0.03 & $81 / 124$ & 95.1 & -0.21 & 19.03 & 18.48 & 17.57 & -21.59 & 2.81 & BL Lac & \\
\hline $\mathrm{J} 161826.93+081950.7$ & 0.4459 & 0.33 & $150 / 119$ & 130.7 & 0.08 & 17.30 & 17.02 & 16.78 & -24.19 & 2.52 & QSO & \\
\hline $\mathrm{J} 161902.49+303051.5$ & 1.2878 & 0.21 & $37 /-$ & - & 一 & 16.95 & 16.84 & 16.52 & -26.46 & 1.94 & QSO & 1,2 \\
\hline $\mathrm{J} 162358.25+074130.5$ & 1.2970 & 0.55 & $170 / 149$ & 100.1 & -0.32 & 17.89 & 18.01 & 17.59 & -25.35 & 3.07 & QSO & \\
\hline $\mathrm{J} 162711.89+314359.3$ & 0.7324 & 0.33 & $36 / 76$ & 127.9 & 0.42 & 20.92 & 20.34 & 20.10 & -21.83 & 3.26 & Sy 1 & \\
\hline $\mathrm{J} 163624.31+471535.9$ & 0.8232 & 0.08 & $5 /-$ & 19.1 & 1.08 & 19.12 & 18.79 & 18.93 & -23.64 & 1.80 & QSO & \\
\hline J163726.66+454748.9 & 0.1922 & 0.12 & $11 / 20$ & 45.3 & 0.66 & 19.78 & 19.01 & 17.83 & -20.32 & 2.14 & BL Lac? & \\
\hline $\mathrm{J} 163856.53+433512.5$ & 0.3392 & 1.21 & $5 / 50$ & 191.8 & 1.08 & 18.48 & 18.45 & 18.27 & -22.22 & 1.60 & Sy1 & 2 \\
\hline $\mathrm{J} 170123.97+385136.9$ & 1.1132 & 0.13 & $49 / 61$ & 198.5 & 0.95 & 18.84 & 18.95 & 18.66 & -24.13 & 2.89 & QSO & 2 \\
\hline $\mathrm{J} 171322.58+325627.9$ & 0.1014 & 0.22 & $25 /-$ & - & - & 18.00 & 17.77 & 16.91 & -20.27 & 1.98 & Sy1 & 1 \\
\hline $\mathrm{J} 172010.03+263732.0$ & 0.1592 & 0.14 & $7 /-$ & - & - & 20.78 & 19.32 & 18.11 & -19.49 & 2.06 & LINERs & 3 \\
\hline $\mathrm{J} 172242.16+281500.0$ & 0.9468 & 0.12 & $150 / 224$ & 249.4 & 0.09 & 18.20 & 18.03 & 18.01 & -24.70 & 2.98 & QSO & 1,2 \\
\hline $\mathrm{J} 205456.85+001537.7$ & 0.1508 & 0.32 & $38 / 65$ & 56.5 & -0.11 & 19.07 & 18.45 & 17.52 & -20.38 & 2.44 & BL Lac? & 2 \\
\hline
\end{tabular}


Notes: (1): SDSS name - Jhhmmss.s+ddmmss.s

(2): $\mathrm{z}-$ redshift

(3): $\Delta_{r o}-$ the distance between the position of radio and optical in units of arcsec

(4): $F_{6 \mathrm{~cm}}$ - flux of $4.85 \mathrm{GHz}$ core and total in units of mJy

(5): $F_{20} \mathrm{~cm}$ - flux of NVSS $1.40 \mathrm{GHz}$ in units of $\mathrm{mJy}$

(6): $\alpha_{r}$ - radio spectral index between $4.85 \mathrm{GHz}$ and $1.40 \mathrm{GHz}$

$$
\alpha_{r}=-\frac{\log \left(F_{4.85 G H z} / F_{1.4 G H z}\right)}{\log \left(\nu_{4.85 G H z} / \nu_{1.4 G H z}\right)}
$$

(7): $u$ - Galactic extinction corrected psf magnitude of SDSS u band

(8): $g$ - Galactic extinction corrected psf magnitude of SDSS $g$ band

(9): $i$ - Galactic extinction corrected psf magnitude of SDSS $i$ band

(10): $M_{B}$ - Galactic extinction corrected B band absolute magnitude

(11): $\lg R-\log$ radio loudness, radio loudness follow 1989

(12): SpecClass — SDSS spectral class

(13): ref — reference about source spectral identification

No reference suggests that it is firstly identified by our work.

1:Laurent-Muehleisen et al. 1998

2:Turriziani et al. 2007

3:Crawford et al. 1999 
Table 3: SDSS Narrow Emission Line Parameters

\begin{tabular}{|c|c|c|c|c|c|c|c|c|}
\hline SDSS name & $\begin{array}{l}\mathrm{F}(H \beta) \\
\lambda 4861\end{array}$ & $\mathrm{~F}([\mathrm{OIII}]$ & $\mathrm{F}(H \alpha)$ & $\mathrm{F}([\mathrm{NII}])$ & $\begin{array}{r}\mathrm{F}([\mathrm{SII}]) \\
\lambda 6716 / \lambda 6731\end{array}$ & $\mathrm{~F}([\mathrm{OI}])$ & $\begin{array}{r}\mathrm{F}([\mathrm{OII}]) \\
\lambda 3727\end{array}$ & SpecClass \\
\hline (1) & $\begin{array}{r}14001 \\
(2)\end{array}$ & (3) & $\begin{array}{r}\lambda 6563 \\
(4)\end{array}$ & $\begin{array}{r}\lambda 6583 \\
(5)\end{array}$ & $\begin{array}{r}\lambda 6716 / \lambda 6731 \\
(6)\end{array}$ & (7) & $\begin{array}{r}\lambda 3 / 2 r \\
(8)\end{array}$ & (9) \\
\hline J074906.50+451033.9 & 308 & 3557 & 1600 & 1026 & $213 / 143$ & 86 & 562 & $\overline{\text { Sy1 }}$ \\
\hline J075244.19+455657.3 & 54 & 899 & 282 & 643 & $205 / 202$ & 233 & 362 & Sy1 \\
\hline J080131.96+473616.0 & 163 & 1292 & 228 & 1251 & $120 / 0$ & 31 & 0 & Sy1 \\
\hline $\mathrm{J} 084203.73+401831.3$ & 75 & 595 & 222 & 115 & $44 / 57$ & 23 & 29 & Sy1 \\
\hline J085004.65+403607.7 & 14 & 64 & 66 & 124 & $26 / 25$ & 37 & 50 & LINER \\
\hline J085348.18+065447.1 & 17 & 354 & 88 & 108 & $38 / 38$ & 49 & 82 & Sy1 \\
\hline J091133.85+442250.1 & 78 & 325 & 421 & 229 & $46 / 18$ & 7 & 102 & Sy1 \\
\hline J092414.70+030900.8 & 87 & 624 & 420 & 315 & $76 / 72$ & 104 & 147 & Sy1 \\
\hline J093200.08+553347.4 & 99 & 399 & 439 & 206 & $55 / 51$ & 13 & 44 & Sy1 \\
\hline J093430.68+030545.3 & 35 & 108 & 180 & 213 & $97 / 112$ & 42 & 195 & LINER \\
\hline $\mathrm{J} 111421.76+582319.8$ & 25 & 23 & 93 & 117 & $48 / 35$ & 30 & 104 & LINER \\
\hline J111908.94+090022.8 & 9 & 17 & 47 & 75 & $16 / 0$ & 9 & 20 & LINER \\
\hline J113255.96+051539.6 & 33 & 261 & 71 & 154 & $49 / 58$ & 39 & 100 & LINER \\
\hline $\mathrm{J} 113518.79+125311.1$ & 22 & 43 & 66 & 106 & $48 / 40$ & 24 & 95 & LINER \\
\hline $\mathrm{J} 115323.95+583138.4$ & 75 & 630 & 203 & 99 & $51 / 50$ & 101 & 168 & Sy1 \\
\hline $\mathrm{J} 115409.28+023815.1$ & 19 & 155 & 91 & 73 & $29 / 28$ & 33 & 130 & Sy1 \\
\hline $\mathrm{J} 115727.60+431806.3$ & 134 & 1417 & 402 & 207 & $88 / 79$ & 41 & 287 & Sy1 \\
\hline $\mathrm{J} 122313.21+540906.5$ & 352 & 2595 & 1490 & 822 & $319 / 266$ & 106 & 782 & Sy1 \\
\hline $\mathrm{J} 132419.67+041907.0$ & 32 & 7 & 99 & 99 & $49 / 39$ & 15 & 99 & LINER \\
\hline $\mathrm{J} 133437.49+563147.9$ & 12 & 316 & 67 & 90 & $23 / 21$ & 8 & 48 & Sy1 \\
\hline $\mathrm{J} 134545.35+533252.2$ & 27 & 342 & 90 & 130 & $61 / 55$ & 52 & 87 & Sy1 \\
\hline $\mathrm{J} 134617.54+622045.4$ & 121 & 1332 & 426 & 213 & $106 / 93$ & 72 & 583 & Sy1 \\
\hline $\mathrm{J} 144542.78+390921.4$ & 13 & 146 & 83 & 66 & $25 / 18$ & 10 & 31 & Sy2 \\
\hline $\mathrm{J} 150117.97+545518.2$ & 56 & 779 & 218 & 326 & $55 / 67$ & 45 & 173 & Sy2 \\
\hline $\mathrm{J} 150324.77+475829.6$ & 54 & 180 & 140 & 162 & $40 / 68$ & 12 & 0 & LINER \\
\hline $\mathrm{J} 151838.90+404500.2$ & 50 & 185 & 252 & 352 & $156 / 130$ & 71 & 292 & LINER \\
\hline $\mathrm{J} 151913.35+362343.4$ & 43 & 300 & 159 & 104 & $34 / 40$ & 34 & 111 & Sy1 \\
\hline $\mathrm{J} 153253.78+302059.3$ & 248 & 145 & 880 & 653 & $0 / 0$ & 216 & 855 & LINER \\
\hline $\mathrm{J} 160239.61+264606.0$ & 57 & 119 & 277 & 439 & $0 / 0$ & 91 & 273 & LINER \\
\hline $\mathrm{J} 164442.53+261913.2$ & 331 & 164 & 1065 & 242 & $0 / 13$ & 0 & 31 & Sy1 \\
\hline $\mathrm{J} 171322.58+325627.9$ & 107 & 1069 & 521 & 460 & $161 / 134$ & 57 & 261 & Sy1 \\
\hline $\mathrm{J} 172010.03+263732.0$ & 91 & 38 & 324 & 281 & $128 / 102$ & 87 & 272 & LINER \\
\hline
\end{tabular}

Notes: (1): SDSS name — Jhhmmss.s+ddmmss.s

(2)-(8): Narrow emission line flux in units of $10^{-17} \mathrm{erg} \mathrm{s}^{-1} \mathrm{~cm}^{-2}$

(9): SpecClass — SDSS spectral class

Table 4: SDSS Broad Emission Line Parameters

\begin{tabular}{cccrrrrc}
\hline $\begin{array}{c}\text { SDSS name } \\
(1)\end{array}$ & $\mathrm{Z}$ & line & flux & FWHM & $\lg L_{B L R}$ & $\lg M_{B H}$ & SpecClass \\
J000132.83+145608.0 & 0.3989 & $H \beta$ & 535 & 3175 & 43.88 & 8.0 & Sy1 \\
J011354.50+132452.4 & 0.6854 & $H \beta$ & 563 & 3699 & 44.47 & 8.5 & QSO \\
J073320.84+390505.2 & 0.6638 & $H \beta$ & 496 & 3471 & 44.38 & 7.5 & QSO \\
J074906.50+451033.9 & 0.1922 & $H \alpha$ & 5462 & 3779 & 43.62 & 8.3 & QSO \\
& & $H \beta$ & 7535 & 4840 & 44.30 & 8.6 & \\
J075244.19+455657.3 & 0.0518 & $H \alpha$ & 4058 & 3067 & 42.27 & 7.5 & Sy1
\end{tabular}




\begin{tabular}{|c|c|c|c|c|c|c|c|}
\hline $\begin{array}{c}\text { SDSS name } \\
\text { (1) }\end{array}$ & $\begin{array}{c}\mathrm{Z} \\
(2)\end{array}$ & $\begin{array}{l}\text { line } \\
(3)\end{array}$ & $\begin{array}{r}\text { flux } \\
(4)\end{array}$ & $\begin{array}{r}\text { FWHM } \\
(5)\end{array}$ & $\begin{array}{l}l g L_{B L R} \\
\text { (6) }\end{array}$ & $\begin{array}{c}\lg M_{B H} \\
\text { (7) }\end{array}$ & $\begin{array}{c}\text { SpecClass } \\
\text { (8) }\end{array}$ \\
\hline J075448.86+303355.1 & 0.7955 & $H \beta$ & 1798 & 8502 & 45.13 & 8.6 & QSO \\
\hline \multirow[t]{2}{*}{ J080131.96+473616.0 } & 0.1569 & $H \beta$ & 11568 & 7287 & 44.29 & 9.0 & QSO \\
\hline & & $H \alpha$ & 9426 & 6319 & 43.66 & 8.7 & \\
\hline J080644.42+484149.2 & 0.3700 & $H \beta$ & 1076 & 14698 & 44.11 & 9.5 & QSO \\
\hline J081058.99+413402.7 & 0.5067 & $H \beta$ & 842 & 3483 & 44.32 & 8.3 & QSO \\
\hline J081100.60+571412.5 & 0.6110 & $H \beta$ & 2226 & 4568 & 44.94 & 8.0 & QSO \\
\hline J081432.11+560956.6 & 0.5094 & $H \beta$ & 782 & 2300 & 44.30 & 8.0 & QSO \\
\hline $\mathrm{J} 081902.32+322637.2$ & 0.6512 & $H \beta$ & 139 & 17188 & 43.81 & 9.4 & Sy1 \\
\hline J081916.61+264203.2 & 0.5261 & $H \beta$ & 368 & 6880 & 44.00 & 8.7 & QSO \\
\hline \multirow[t]{2}{*}{ J084203.73+401831.3 } & 0.1516 & $H \beta$ & 4188 & 8810 & 43.82 & 8.8 & Sy1 \\
\hline & & $H \alpha$ & 3358 & 7774 & 43.18 & 8.7 & \\
\hline J084650.00+064149.0 & 0.6161 & $H \beta$ & 2751 & 7836 & 45.04 & 9.5 & QSO \\
\hline J085039.95+543753.3 & 0.3673 & $H \beta$ & 1079 & 12595 & 44.10 & 9.3 & Sy1 \\
\hline J085348.18+065447.1 & 0.2234 & $H \alpha$ & 704 & 3813 & 42.87 & 7.9 & Sy1 \\
\hline J085830.61+080422.8 & 0.4549 & $H \beta$ & 1132 & 6634 & 44.34 & 8.9 & QSO \\
\hline J090745.28+532421.4 & 0.7113 & $H \beta$ & 1074 & 7213 & 44.79 & 9.3 & QSO \\
\hline J090835.85+415046.3 & 0.7337 & $H \beta$ & 867 & 3545 & 44.73 & 8.6 & QSO \\
\hline J090924.66+274402.8 & 0.3342 & $H \beta$ & 454 & 9815 & 43.63 & 8.8 & Sy1 \\
\hline J090924.68+521632.6 & 0.4102 & $H \beta$ & 1507 & 10542 & 44.36 & 9.3 & QSO \\
\hline \multirow{2}{*}{ J091133.85+442250.1 } & 0.2976 & $H \beta$ & 873 & 2861 & 43.80 & 7.8 & Sy1 \\
\hline & & $H \alpha$ & 734 & 2688 & 43.18 & 7.8 & \\
\hline \multirow{2}{*}{ J092414.70+030900.8 } & 0.1280 & $H \alpha$ & 460 & 6140 & 42.16 & 8.0 & Sy1 \\
\hline & & $H \beta$ & 2087 & 6148 & 43.36 & 7.5 & \\
\hline \multirow[t]{2}{*}{ J093200.08+553347.4 } & 0.2657 & $H \beta$ & 2104 & 4531 & 44.06 & 8.4 & QSO \\
\hline & & $H \alpha$ & 1738 & 4198 & 43.44 & 8.3 & \\
\hline \multirow[t]{2}{*}{ J093712.33+500852.1 } & 0.2756 & $H \alpha$ & 123 & 2922 & 42.32 & 7.5 & Sy1 \\
\hline & & $H \beta$ & 543 & 2923 & 43.51 & 6.9 & \\
\hline J094857.31+002225.5 & 0.5846 & $H \beta$ & 301 & 1850 & 44.03 & 7.6 & NLSy1 \\
\hline J095855.10+423704.0 & 0.6641 & $H \beta$ & 415 & 2817 & 44.30 & 7.3 & QSO \\
\hline $\mathrm{J} 101447.77+442133.3$ & 0.7956 & $H \beta$ & 320 & 5468 & 44.38 & 8.8 & QSO \\
\hline $\mathrm{J} 102044.32+492046.2$ & 0.3899 & $H \beta$ & 898 & 4396 & 44.08 & 7.5 & QSO \\
\hline J102106.04+452331.8 & 0.3644 & $H \beta$ & 1086 & 7669 & 44.09 & 8.9 & QSO \\
\hline $\mathrm{J} 102235.57+454105.4$ & 0.7432 & $H \beta$ & 424 & 16214 & 44.43 & 9.7 & Sy1 \\
\hline J102237.44+393150.1 & 0.6036 & $H \beta$ & 473 & 4747 & 44.26 & 8.6 & QSO \\
\hline $\mathrm{J} 102504.20+414332.6$ & 0.6818 & $H \beta$ & 706 & 6159 & 44.56 & 9.0 & QSO \\
\hline \multirow[t]{2}{*}{$\mathrm{J} 102738.53+605016.5$} & 0.3320 & $H \beta$ & 1312 & 23649 & 44.08 & 9.9 & QSO \\
\hline & & $H \alpha$ & 727 & 15006 & 43.28 & 9.4 & \\
\hline J103024.95+551622.7 & 0.4345 & $H \beta$ & 3828 & 2244 & 44.82 & 8.3 & QSO \\
\hline $\mathrm{J} 103144.75+602030.4$ & 1.2303 & MgII & 465 & 3041 & 44.82 & - & QSO \\
\hline $\mathrm{J} 103214.53+635950.3$ & 0.5562 & $H \beta$ & 536 & 5623 & 44.23 & 8.7 & QSO \\
\hline $\mathrm{J} 104334.81+343232.5$ & 0.7330 & $H \beta$ & 197 & 6264 & 44.08 & 8.7 & Sy1 \\
\hline J104410.67+532220.5 & 1.9008 & MgII & 236 & 2591 & 44.99 & - & QSO \\
\hline J105431.89+385521.6 & 1.3661 & MgII & 352 & 2452 & 44.81 & - & QSO \\
\hline J110409.63+122157.5 & 0.5907 & $H \beta$ & 1791 & 5493 & 44.81 & 8.1 & QSO \\
\hline J114510.39+011056.2 & 0.6261 & $H \beta$ & 264 & 6572 & 44.04 & 8.7 & QSO \\
\hline J114803.17+565411.4 & 0.4510 & $H \beta$ & 1979 & 6159 & 44.57 & 9.0 & QSO \\
\hline J115227.48+320959.4 & 0.3747 & $H \beta$ & 527 & 5080 & 43.81 & 8.3 & Sy1 \\
\hline J115232.86+493938.7 & 1.0929 & MgII & 1991 & 3801 & 45.33 & - & QSO \\
\hline \multirow[t]{2}{*}{ J115323.95+583138.4 } & 0.2024 & $H \alpha$ & 1203 & 6066 & 43.01 & 8.4 & Sy1 \\
\hline & & $H \beta$ & 4592 & 6066 & 44.14 & 7.8 & \\
\hline J115326.70+361726.3 & 1.3580 & MgII & 371 & 2034 & 44.83 & - & QSO \\
\hline
\end{tabular}




\begin{tabular}{|c|c|c|c|c|c|c|c|}
\hline $\begin{array}{l}\text { SDSS name } \\
\text { (1) }\end{array}$ & $\begin{array}{c}\mathrm{Z} \\
\text { (2) }\end{array}$ & $\begin{array}{l}\text { line } \\
(3)\end{array}$ & $\begin{array}{r}\text { flux } \\
\text { (4) }\end{array}$ & $\begin{array}{r}\text { FWHM } \\
(5)\end{array}$ & $\begin{array}{c}l g L_{B L R} \\
\text { (6) }\end{array}$ & $\begin{array}{c}\lg M_{B H} \\
\text { (7) }\end{array}$ & $\begin{array}{c}\text { SpecClass } \\
\text { (8) }\end{array}$ \\
\hline $\mathrm{J} 115409.28+023815.1$ & 0.2106 & $H \alpha$ & 602 & 3278 & 42.75 & 7.8 & Sy1 \\
\hline $\mathrm{J} 115700.59+324457.8$ & 0.4862 & $H \beta$ & 898 & 2318 & 44.31 & 8.0 & QSO \\
\hline \multirow[t]{2}{*}{ J115727.60+431806.3 } & 0.2300 & $H \alpha$ & 670 & 2867 & 42.88 & 7.7 & Sy 1 \\
\hline & & $H \beta$ & 952 & 3274 & 43.58 & 7.8 & \\
\hline $\mathrm{J} 120335.39+451049.5$ & 1.0766 & MgII & 1230 & 3726 & 45.10 & - & QSO \\
\hline J120436.17+485653.9 & 0.4509 & $H \beta$ & 965 & 3422 & 44.26 & 8.3 & QSO \\
\hline $\mathrm{J} 121509.95+462715.1$ & 0.7201 & $H \beta$ & 2276 & 4550 & 45.13 & 9.1 & QSO \\
\hline \multirow[t]{2}{*}{$\mathrm{J} 122313.21+540906.5$} & 0.1559 & $H \beta$ & 3118 & 6708 & 43.72 & 8.5 & Sy1 \\
\hline & & $H \alpha$ & 2221 & 5111 & 43.03 & 8.3 & \\
\hline $\mathrm{J} 122424.23+401510.5$ & 0.4164 & $H \beta$ & 1879 & 5500 & 44.47 & 8.8 & QSO \\
\hline $\mathrm{J} 122506.50+483435.1$ & 0.6468 & $H \beta$ & 534 & 5092 & 44.38 & 8.7 & QSO \\
\hline $\mathrm{J} 123157.08+542028.7$ & 0.5156 & $H \beta$ & 337 & 6794 & 43.94 & 7.9 & QSO \\
\hline \multirow[t]{2}{*}{$\mathrm{J} 123807.76+532556.0$} & 0.3475 & $H \alpha$ & 2021 & 14772 & 43.77 & 9.6 & QSO \\
\hline & & $H \beta$ & 2206 & 13890 & 44.35 & 9.6 & \\
\hline $\mathrm{J} 123819.62+412420.5$ & 0.4980 & $H \beta$ & 1041 & 9322 & 44.40 & 9.2 & QSO \\
\hline $\mathrm{J} 124139.72+493405.5$ & 0.4739 & $H \beta$ & 1785 & 8082 & 44.58 & 9.2 & QSO \\
\hline \multirow[t]{2}{*}{$\mathrm{J} 125223.78+645137.9$} & 0.3119 & $H \beta$ & 1579 & 4217 & 44.10 & 8.4 & QSO \\
\hline & & $H \alpha$ & 1330 & 4322 & 43.48 & 8.3 & \\
\hline $\mathrm{J} 131211.14+480925.3$ & 0.7149 & $H \beta$ & 2411 & 4673 & 45.14 & 8.1 & QSO \\
\hline $\mathrm{J} 132631.44+473755.8$ & 0.6822 & $H \beta$ & 838 & 5025 & 44.63 & 8.9 & QSO \\
\hline $\mathrm{J} 133245.24+472222.6$ & 0.6694 & $H \beta$ & 499 & 3372 & 44.39 & 8.4 & QSO \\
\hline \multirow[t]{2}{*}{ J133437.49+563147.9 } & 0.3428 & $H \beta$ & 784 & 3662 & 43.89 & 8.1 & QSO \\
\hline & & $H \alpha$ & 633 & 3397 & 43.25 & 8.0 & \\
\hline $\mathrm{J} 133655.51+654116.0$ & 0.4368 & $H \beta$ & 1196 & 15462 & 44.32 & 9.6 & QSO \\
\hline \multirow[t]{2}{*}{$\mathrm{J} 134545.35+533252.2$} & 0.1354 & $H \beta$ & 2135 & 5413 & 43.42 & 8.2 & Sy1 \\
\hline & & $H \alpha$ & 2121 & 5253 & 42.87 & 8.2 & \\
\hline \multirow[t]{2}{*}{$\mathrm{J} 134617.54+622045.4$} & 0.1165 & $H \alpha$ & 2234 & 5721 & 42.75 & 8.3 & Sy1 \\
\hline & & $H \beta$ & 8607 & 5724 & 43.88 & 7.7 & \\
\hline $\mathrm{J} 134751.58+283629.7$ & 0.7399 & $H \beta$ & 1524 & 5358 & 44.98 & 9.1 & QSO \\
\hline $\mathrm{J} 135229.02+490823.0$ & 0.3966 & $H \beta$ & 441 & 4704 & 43.79 & 8.3 & Sy1 \\
\hline J135305.54+044338.6 & 0.5234 & $H \beta$ & 1098 & 10857 & 44.47 & 9.4 & QSO \\
\hline J135341.72+431052.5 & 1.1136 & MgII & 494 & 2291 & 44.74 & - & QSO \\
\hline $\mathrm{J} 141159.73+423950.3$ & 0.8865 & MgII & 1838 & 4069 & 45.07 & - & QSO \\
\hline J141446.64+392818.6 & 0.6571 & $H \beta$ & 366 & 4044 & 44.24 & 8.4 & QSO \\
\hline $\mathrm{J} 141628.66+124213.5$ & 0.3345 & $H \beta$ & 3353 & 9439 & 44.50 & 9.3 & QSO \\
\hline J141740.44+381821.1 & 0.4494 & $H \beta$ & 804 & 2947 & 44.18 & 8.1 & QSO \\
\hline $\mathrm{J} 142020.67+462440.8$ & 1.2450 & MgII & 327 & 9294 & 44.68 & - & QSO \\
\hline $\mathrm{J} 142106.03+385522.7$ & 0.4888 & MgII & 954 & 3426 & 44.15 & - & QSO \\
\hline \multirow[t]{2}{*}{$\mathrm{J} 142314.19+505537.3$} & 0.2759 & $H \beta$ & 1667 & 1270 & 44.00 & 7.3 & QSO \\
\hline & & $H \alpha$ & 1352 & 2848 & 43.37 & 7.9 & \\
\hline $\mathrm{J} 142606.19+402432.0$ & 0.6639 & $H \beta$ & 421 & 4168 & 44.31 & 8.5 & Sy1 \\
\hline $\mathrm{J} 143726.14+504555.8$ & 0.7833 & $H \beta$ & 2439 & 5826 & 45.25 & 9.4 & QSO \\
\hline $\mathrm{J} 143942.83+582759.2$ & 0.4250 & $H \beta$ & 969 & 4575 & 44.20 & 8.5 & QSO \\
\hline $\mathrm{J} 145224.68+452223.6$ & 0.4671 & $H \beta$ & 4419 & 6566 & 44.96 & 9.3 & QSO \\
\hline $\mathrm{J} 145247.37+473529.1$ & 1.1582 & MgII & 643 & 6007 & 44.90 & - & QSO \\
\hline $\mathrm{J} 145958.43+333701.8$ & 0.6448 & $H \beta$ & 3185 & 4704 & 45.16 & 8.1 & QSO \\
\hline $\mathrm{J} 150455.56+564920.2$ & 0.3589 & $H \beta$ & 3085 & 6036 & 44.53 & 8.9 & QSO \\
\hline \multirow[t]{2}{*}{$\mathrm{J} 151017.82+422155.0$} & 0.4873 & $H \beta$ & 724 & 1880 & 44.22 & 7.7 & Sy1 \\
\hline & & MgII & 205 & 5621 & 43.48 & - & \\
\hline J151830.93+483214.4 & 0.5757 & $H \beta$ & 951 & 8909 & 44.51 & 9.3 & QSO \\
\hline $\mathrm{J} 151844.76+461855.1$ & 0.8853 & MgII & 449 & 3651 & 44.45 & - & QSO \\
\hline
\end{tabular}




\begin{tabular}{|c|c|c|c|c|c|c|c|}
\hline $\begin{array}{c}\text { SDSS name } \\
\text { (1) }\end{array}$ & $\begin{array}{c}\mathrm{Z} \\
(2)\end{array}$ & $\begin{array}{l}\text { line } \\
(3)\end{array}$ & $\begin{array}{r}\text { flux } \\
\text { (4) }\end{array}$ & $\begin{array}{r}\text { FWHM } \\
(5)\end{array}$ & $\begin{array}{c}\lg L_{B L R} \\
\text { (6) }\end{array}$ & $\begin{array}{c}\lg M_{B H} \\
\text { (7) }\end{array}$ & $\begin{array}{c}\text { SpecClass } \\
\text { (8) }\end{array}$ \\
\hline \multirow[t]{2}{*}{ J151913.35+362343.4 } & 0.2857 & $H \alpha$ & 404 & 8218 & 42.88 & 8.6 & Sy1 \\
\hline & & $H \beta$ & 1642 & 8222 & 44.03 & 8.1 & \\
\hline $\mathrm{J} 152556.22+591659.5$ & 0.9551 & MgII & 1421 & 8940 & 45.03 & - & QSO \\
\hline $\mathrm{J} 153102.48+435637.6$ & 0.4520 & $H \beta$ & 908 & 2330 & 44.24 & 7.9 & QSO \\
\hline $\mathrm{J} 154232.03+493842.5$ & 0.5897 & $H \beta$ & 381 & 5499 & 44.14 & 7.8 & QSO \\
\hline J160317.91+090037.9 & 0.4883 & $H \beta$ & 1497 & 9389 & 44.54 & 9.3 & QSO \\
\hline $\mathrm{J} 160658.30+271705.5$ & 0.9337 & MgII & 669 & 3041 & 44.68 & - & QSO \\
\hline J160813.79+292126.3 & 1.2005 & MgII & 362 & 6339 & 44.69 & - & QSO \\
\hline $\mathrm{J} 160822.16+401217.9$ & 0.6281 & $H \beta$ & 216 & 3274 & 43.96 & 8.1 & Sy1 \\
\hline J161826.93+081950.7 & 0.4455 & $H \beta$ & 4067 & 19802 & 44.87 & 10.2 & QSO \\
\hline J161902.49+303051.5 & 1.2875 & MgII & 1209 & 3351 & 45.29 & 一 & QSO \\
\hline $\mathrm{J} 162111.27+374604.9$ & 1.2728 & MgII & 175 & 3159 & 44.43 & - & QSO \\
\hline $\mathrm{J} 162229.31+400643.5$ & 0.6878 & $H \beta$ & 814 & 3545 & 44.63 & 8.5 & QSO \\
\hline $\mathrm{J} 162358.25+074130.5$ & 1.2973 & MgII & 648 & 3137 & 45.02 & - & QSO \\
\hline J162711.89+314359.3 & 0.7324 & $H \beta$ & 490 & 13816 & 44.48 & 9.6 & Sy1 \\
\hline J163624.31+471535.9 & 0.8233 & $H \beta$ & 441 & 3570 & 44.56 & 7.6 & QSO \\
\hline \multirow[t]{2}{*}{$\mathrm{J} 163856.53+433512.5$} & 0.3390 & $H \beta$ & 1163 & 11516 & 44.05 & 9.2 & Sy 1 \\
\hline & & $H \alpha$ & 978 & 10092 & 43.43 & 9.1 & \\
\hline J164054.16+314329.9 & 0.9580 & MgII & 602 & 5985 & 44.66 & - & QSO \\
\hline \multirow[t]{2}{*}{$\mathrm{J} 164442.53+261913.2$} & 0.1443 & $H \alpha$ & 980 & 1535 & 42.60 & 7.0 & NLSy1 \\
\hline & & $H \beta$ & 2771 & 1544 & 43.59 & 6.4 & \\
\hline $\mathrm{J} 165005.47+414032.4$ & 0.5848 & $H \beta$ & 1296 & 5240 & 44.66 & 8.9 & QSO \\
\hline $\mathrm{J} 170112.38+353353.4$ & 0.5011 & $H \beta$ & 495 & 2256 & 44.08 & 7.8 & QSO \\
\hline J170123.97+385136.9 & 1.1125 & MgII & 435 & 4625 & 44.68 & - & QSO \\
\hline \multirow[t]{2}{*}{$\mathrm{J} 171322.58+325627.9$} & 0.1013 & $H \alpha$ & 1033 & 3933 & 42.29 & 7.7 & Sy1 \\
\hline & & $H \beta$ & 3786 & 3933 & 43.40 & 7.1 & \\
\hline $\mathrm{J} 172242.16+281500.0$ & 0.9447 & MgII & 663 & 2762 & 44.69 & - & QSO \\
\hline
\end{tabular}

Notes: (1): SDSS name - Jhhmmss.s+ddmmss.s

(2): $\mathrm{z}$ - redshift of emission line

(3): line — broad emission line

(4): flux - broad emission line flux in units of $10^{-17} \mathrm{erg} \mathrm{s}^{-1} \mathrm{~cm}^{-2}$

(5): FWHM - FWHM for broad emission line in units of $\mathrm{km} \mathrm{s}^{-1}$

(6): $\lg L_{B L R}-\log$ broad emission line region luminosity in units of erg s${ }^{-1}$

(7): $\lg M_{B H}-\log$ Black Hole mass in units of Solar mass

(8): SpecClass — SDSS spectral class

Table 5: Narrow line region luminosities and black Hole masses of the identified LINERs

\begin{tabular}{cccc}
\hline $\begin{array}{c}\text { SDSS name } \\
(1)\end{array}$ & $\begin{array}{c}\lg L_{N L R} \\
(2)\end{array}$ & $\begin{array}{c}\sigma_{*} \\
(3)\end{array}$ & $\begin{array}{c}\lg M_{B H} \\
(4)\end{array}$ \\
\hline $\mathrm{J} 085004.65+403607.7$ & 42.21 & $412 \pm 22$ & 9.4 \\
$\mathrm{~J} 093430.68+030545.3$ & 42.53 & $393 \pm 16$ & 9.3 \\
$\mathrm{~J} 111421.76+582319.8$ & 42.11 & & \\
$\mathrm{~J} 111908.94+090022.8$ & 41.96 & $315 \pm 29$ & 8.9 \\
$\mathrm{~J} 113255.96+051539.6$ & 41.73 & $231 \pm 7$ & 8.4 \\
$\mathrm{~J} 113518.79+125311.1$ & 42.10 & & \\
$\mathrm{~J} 132419.67+041907.0$ & 42.29 & $375 \pm 38$ & 9.2 \\
$\mathrm{~J} 150324.77+475829.6$ & 42.68 & & \\
$\mathrm{~J} 151838.90+404500.2$ & 41.55 & $241 \pm 7$ & 8.5
\end{tabular}




\begin{tabular}{cccc}
\hline $\begin{array}{c}\text { SDSS name } \\
(1)\end{array}$ & $\begin{array}{c}\lg L_{N L R} \\
(2)\end{array}$ & $\begin{array}{c}\sigma_{*} \\
(3)\end{array}$ & $\begin{array}{c}\lg M_{B H} \\
(4)\end{array}$ \\
\hline $\mathrm{J} 153253.78+302059.3$ & 43.57 & & \\
$\mathrm{~J} 160239.61+264606.0$ & 43.15 & $429 \pm 87$ & 9.5 \\
$\mathrm{~J} 172010.03+263732.0$ & 42.26 & $364 \pm 19$ & 9.2 \\
\hline
\end{tabular}

Notes: (1): SDSS name - Jhhmmss.s+ddmmss.s

(2): $\lg L_{N L R}-\log$ narrow emission line region luminosity in Unit: erg s$^{-1}$

(3): $\sigma_{*}$ - velocity dispersion and velocity dispersion error of the host galaxy in Unit: $\mathrm{km} \mathrm{s}^{-1}$

(4): $\lg M_{B H}-\log$ Black Hole mass in Unit: Solar mass

Table 6: Comparison with previous RGB and ROXA identifications

\begin{tabular}{cccc}
\hline $\begin{array}{c}\text { SDSS name } \\
(1)\end{array}$ & $\begin{array}{c}\text { our ID } \\
(2)\end{array}$ & $\begin{array}{c}\text { previous ID } \\
(3)\end{array}$ & $\begin{array}{c}\text { ref } \\
(4)\end{array}$ \\
\hline J083353.88+422401.8 & BL Lac & FSRQ & 2 \\
J111908.94+090022.8 & LINERs & BL Lac & 2 \\
J113518.79+125311.1 & LINERs & Radio Galaxy & 2 \\
J115409.28+023815.1 & FSRQ & FSRQ/BL Lac & 2 \\
J120303.50+603119.1 & BL Lac & LINERs & 3,2 \\
J124834.30+512807.8 & BL Lac & Star & $1,2,4$ \\
J132419.67+041907.0 & LINERs & SSRQ & 2 \\
J141149.43+524900.1 & Galaxy & BL Lac & 2 \\
J142730.27+540923.7 & Galaxy & BL Lac & 1 \\
J150117.97+545518.2 & Seyfert 2 & SSRQ & 2 \\
J153253.78+302059.3 & LINERs & NELG & 2 \\
\hline
\end{tabular}

Notes: (1): SDSS name — Jhhmmss.s+ddmmss.s

(2): our ID — our SDSS spectral class

(3): previous ID - previous spectral class

(4): ref — reference about source spectral identification

1:Laurent-Muehleisen et al.1998

2:Turriziani et al. 2007

3:Carrillo et al. 1999

4:Collinge et al.2005

Table 7: BL Lac with a featureless continuum

\begin{tabular}{ccccccl}
\hline $\begin{array}{c}\text { SDSS name } \\
(1)\end{array}$ & $\begin{array}{c}u \\
(2)\end{array}$ & $\begin{array}{c}g \\
(3)\end{array}$ & $\begin{array}{c}r \\
(4)\end{array}$ & $\begin{array}{c}i \\
(5)\end{array}$ & $\begin{array}{c}z \\
(6)\end{array}$ & $\begin{array}{c}\text { ref } \\
(7)\end{array}$ \\
\hline J085409.88+440830.2 & 17.33 & 17.02 & 16.81 & 16.67 & 16.55 & 1,2 \\
J092915.43+501336.1 & 17.26 & 16.86 & 16.49 & 16.22 & 16.01 & 2 \\
J100110.20+291137.6 & 19.02 & 18.52 & 18.08 & 17.75 & 17.41 & \\
J103744.30+571155.6 & 17.07 & 16.68 & 16.38 & 16.13 & 15.91 & 1,2 \\
J110124.72+410847.4 & 19.35 & 19.06 & 18.80 & 18.58 & 18.37 & 2 \\
J110748.07+150210.5 & 18.53 & 18.27 & 18.05 & 17.88 & 17.71 & \\
J112453.82+493409.7 & 18.96 & 18.70 & 18.50 & 18.37 & 18.15 & 2 \\
J115124.67+585917.7 & 16.40 & 16.08 & 15.89 & 15.64 & 15.58 & 2 \\
J120922.78+411941.3 & 18.38 & 17.99 & 17.61 & 17.34 & 17.10 & 2 \\
J124700.72+442318.7 & 19.02 & 18.84 & 18.50 & 18.37 & 18.12 & 2 \\
J130145.65+405624.6 & 18.55 & 18.28 & 18.04 & 17.91 & 17.76 & 2 \\
J135120.84+111453.0 & 18.99 & 18.72 & 18.50 & 18.21 & 18.04 & 2
\end{tabular}




\begin{tabular}{ccccccl}
\hline $\begin{array}{c}\text { SDSS name } \\
(1)\end{array}$ & $\begin{array}{c}u \\
(2)\end{array}$ & $\begin{array}{c}g \\
(3)\end{array}$ & $\begin{array}{c}r \\
(4)\end{array}$ & $\begin{array}{c}i \\
(5)\end{array}$ & $\begin{array}{c}z \\
(6)\end{array}$ & $\begin{array}{l}\text { ref } \\
(7)\end{array}$ \\
\hline $\mathrm{J} 141536.80+483030.4$ & 19.61 & 19.18 & 18.74 & 18.36 & 18.11 & 1 \\
$\mathrm{~J} 142607.71+340426.3$ & 17.95 & 17.59 & 17.33 & 17.09 & 16.90 & \\
$\mathrm{~J} 144052.93+061016.1$ & 17.77 & 17.38 & 17.08 & 16.83 & 16.63 & \\
$\mathrm{~J} 144800.58+360831.0$ & 17.32 & 16.96 & 16.75 & 16.50 & 16.41 & 1,2 \\
$\mathrm{~J} 145427.13+512433.7$ & 18.58 & 18.17 & 17.77 & 17.46 & 17.20 & 1,2 \\
$\mathrm{~J} 150947.97+555617.2$ & 18.44 & 18.03 & 17.72 & 17.47 & 17.21 & 2 \\
$\mathrm{~J} 153324.26+341640.3$ & 18.29 & 18.04 & 17.86 & 17.65 & 17.49 & 1,2 \\
$\mathrm{~J} 160218.06+305109.4$ & 18.49 & 18.13 & 17.91 & 17.77 & 17.59 & 1,2 \\
$\mathrm{~J} 161830.59+062211.5$ & 19.25 & 18.84 & 18.46 & 18.16 & 17.97 & \\
$\mathrm{~J} 165249.92+402310.1$ & 18.90 & 18.57 & 18.31 & 18.12 & 18.04 & 1,2 \\
\hline
\end{tabular}

Notes: (1): SDSS name - Jhhmmss.s+ddmmss.s

(2)-(6): Galactic extinction corrected psf magnitude of SDSS

(7): ref — reference about source spectral identification

No reference suggests that it is firstly identified by our work.

1:Laurent-Muehleisen et al. 1998

2:Turriziani et al.2007

\section{Appendix A: SDSS STARLIGHT SPECTRAL MODELING}

The spectra are first corrected for Galactic extinction using the extinction map of Schlegel et al. (1998) and the reddening curve of Fitzpatrick (1999), and transformed into the rest frame using the redshift provided by the SDSS pipeline. Then, host-galaxy starlight and AGN continuum, as well as the optical Fe II emission complex are modeled as

$$
S(\lambda)=A_{\text {host }}\left(E_{B-V}^{\text {host }}, \lambda\right) A(\lambda)+A_{\text {nucleus }}\left(E_{B-V}^{\text {nucleus }}, \lambda\right)\left[b B(\lambda)+c_{\mathrm{b}} C_{\mathrm{b}}(\lambda)+c_{\mathrm{n}} C_{\mathrm{n}}(\lambda)\right]
$$

where $S(\lambda)$ is the observed spectrum. $A(\lambda)=\sum_{i=1}^{6} a_{i} I C_{i}\left(\lambda, \sigma_{*}\right)$ represents the starlight component modeled by our six synthesized galaxy templates, which was built up from the spectral template library of Simple Stellar Populations (SSPs) of Bruzual \& Charlot (2003) using our new method based on the Ensemble Learning Independent Component Analysis (EL-ICA) algorithm. The details of the galaxy templates and their applications are presented in Lu et al. (2006). $A(\lambda)$ is broadened by convolving it with a Gaussian of width $\sigma_{*}$ to match the stellar velocity dispersion of the host galaxy. The un-reddened nuclear continuum is assumed to be $B(\lambda)=\lambda^{-1.7}$ as given in Francis (1996). We modeled the optical Fe II emission, both broad and narrow, using the spectral data of the Fe II multiplets for IZw I in the $\lambda \lambda 3535-7530 \AA$ range provided by Véron-Cetty et al. (2004)(Table A1,A2). We assume that the broad Fe II lines $\left(C_{\mathrm{b}}\right.$ in Eq.(A.1)) have the same profiles as the broad $\mathrm{H} \beta$ line, and the narrow Fe II lines $\left(C_{\mathrm{n}}\right)$, both permitted and forbidden, have the same profiles as the that of the narrow $\mathrm{H} \beta$ component, or of [O III] $\lambda 5007$ if $\mathrm{H} \beta$ is weak. $A_{\text {host }}\left(E_{B-V}^{\text {host }}, \lambda\right)$ and $A_{\text {nucleus }}\left(E_{B-V}^{\text {nucleus }}, \lambda\right)$ are the color excesses due to possible extinction of the host galaxy and the nuclear region, respectively, assuming the extinction curve for the Small Magellanic Cloud of Pei (1992). The fitting is performed by minimizing the $\chi^{2}$ with $E_{B-V}^{\text {host }}, E_{B-V}^{\text {nucleus }}, a_{i}, \sigma_{*}, b, c_{\mathrm{b}}$ and $c_{\mathrm{n}}$ being free parameters. To account for possible errors of the redshifts provided by the SDSS pipeline, in practice, we iterate the procedure with redshifts with a step of $5 \mathrm{~km} \mathrm{~s}^{-1}$ near the SDSS redshift and use the best-fit result.

The emission line spectra are fitted in the following way. As the narrow Balmer lines and the [N II] $\lambda \lambda 6548,6583$ doublet have similar profiles to the [S II] $\lambda \lambda 6716,6731$ doublet lines (Filippenko et al. 1988, Ho et al. 1997, Zhou et al. 2006), we use the [S II] $\lambda \lambda 6716,6731$ doublet lines as a template to fit the narrow lines. If [S II] is weak, [O III] $\lambda 5007$ is used as the template. Each of the [S II] doublet lines is assumed to have the same profile and redshift, and is fitted with as many Gaussians as is statistically justified; generally 1-2 Gaussians are needed. Likewise, the [O III] $\lambda \lambda 4959,5007$ doublet are fitted in a similar way, with the flux ratio of [O III] $\lambda 5007 /[\mathrm{O}$ III $] \lambda 4959$ fixed to the theoretical value of 3 . When a good model of the narrow-line template is achieved, we scale it to fit the narrow Balmer lines and the [N II] $\lambda \lambda 6548,6583$ doublet lines. The flux ratio of the [N II] doublet $\lambda 6583 / \lambda 6548$ is fixed to the theoretical 
value of 2.96. For possible broad $\mathrm{H} \alpha$ and $\mathrm{H} \beta$ lines, we use multiple Gaussians to fit them, as many as is statistically justified. If a broad $\mathrm{H} \beta$ line is too weak to get a reliable fit, we then re-fit it assuming it has the same profile and redshift as a broad $\mathrm{H} \alpha$. If a broad-line emission is detected at the $\geq 5 \sigma$ confidence level, we regard it as genuine.

\section{References}

Abdo, A. A., Ackermann, M., Ajello, M., et al., 2009, ApJ, 699, 976

Baldwin, J. A., Phillips, M. M., Terlevich, R., 1981, PASP, 93, 5

Brinkmann, W., Siebert, J., Reich, W. et al., 1995, A\&AS, 109, 147

Brinkmann, W., Siebert, J., Feigelson, E. D. et al., 1997, A\&A, 323, 739

Bruzual, G., Charlot, S., 2003, MNRAS, 344, 1000

Caccianiga, A., Maccacaro, T., Wolter, A. et al., 1999, ApJ, 513, 51

Caccianiga, A., Maccacaro, T., Wolter, A. et al., 2000, A\&AS, 144, 247

Carrillo R., Masegosa, J., et al., 1999, Rev.Mex.AA, 35, 187

Celotti, A., Fabian, A. C., 1993, MNRAS, 264, 228

Celotti, A., Padovani, P., \& Ghisellini, G. 1997, MNRAS, 286, 415

Costero, R. \& Osterbrock, D.E., 1977, ApJ, 211, 675

Collinge, M. J., Strauss, M. A., Hall, P. B. et al., 2005, AJ, 129, 2542

Crawford, C. S., Allen, S. W., Ebeling, H., Edge, A. C., Fabian, A. C, 199, MNRAS, 306, 857

Dong, X.B., Zhou, H.Y., Wang, T.G. et al., 2005, ApJ, 620, 629

Dressler, A., Shectman, S. A., 1987, AJ, 94, 899

Edge, A. C., Ebeling, H., Bremer, M. et al., 2003, MNRAS, 339, 913

Falcke, H., et al., 2000, ApJ, 542, 197

Filippenko, A. V., Sargent, W.L.W., et al., 1988, ApJ, 324, 134

Fitzpatrick, E. L., 1999, PASP, 111, 63

Fossati, G., Maraschi, L., Celotti, A. et al., 1998, MNRAS, 299, 433

Francis, P. J., Hewett, P. C., Foltz, C. B. et al., 1991, ApJ, 373, 465

Francis, P. J., 1996, Publ.Astron.Soc.Australia, 13, 212

Gaskell, C. M., Sheilds, G. A., Wampler, E. J., 1981, ApJ, 249, 443

Ghisellini, G., Celotti, A., Fossati, G. et al., 1998, MNRAS, 301, 451

Giommi, P., Menna, M. T., \& Padovani, P, 1999, MNRAS, 310, 465

Giommi, P., Piranomonte, S., Perri, M. et al., 2005, A\&A, 434, 385

Greene, J. E., Ho, L. C., 2005, ApJ, 630, 122

Greene, J. E., Ho, L. C., 2007a, ApJ, 670, 92

Greene, J. E., Ho, L. C., 2007b, ApJ, 667, 113

Gregory P. C., Condon J. J., 1991, ApJS, 75. 1011

Gregory P. C., Scott W. K., Douglas K., 1996, ApJS, 103, 427

Heckman T. M., 1980, A\&A, 87, 152

Hine, R.G. \& Longair, M.S., 1979, MNRAS, 188, 111

Ho, L.C., Filippenko, A.V., \& Sargent,W. L.W., 1995, ApJS, 98, 477

Ho, L. C., et al., 1997, ApJs, 112, 315

Ho, L.C., 2008, ARA\&A, 46, 475

Jester, Sebastian, 2005, AJ, 130, 873J

Kauffmann, G., Heckman, T. M., Tremonti, C. et al., 2003, MNRAS, 346, 1055

Kellermann, K. I., Sramek, R., Schmidt, M. et al., 1989, AJ, 98, 1195

Kewley, L. J., Groves, B., Kauffmann, G. et al., 2006, MNRAS, 372, 961 
Kewley, L. J., Dopita, M. A., Sutherland, R. S. et al., 2001, ApJ, 556, 121

Komossa, S., Voges, W., Xu, D. et al.,2006, AJ, 132, 531

Koester, B. P., McKay, T. A., Annis, J. et al., 2007, ApJ, 660, 239

Koski, A.T., 1978, ApJ, 223, 56

Laing, R.A., et al. 1994, 'The physics of Active Galaxies', Bicknell G.V., Dopita, M.A., Quinn P.J. (eds.) ASPC, 54, 201

Landt, H., Padovani, P., Perlman, E. S. et al., 2001, MNRAS, 323, 757

Landt, H., Padovani, P., Giommi, P., 2002, MNRAS, 336, 945

Laurent-Muehleisen, S. A., Kollgaard, R. I., Ryan, P. J. et al., 1997, A\&AS, 122, 235

Laurent-Muehleisen, S. A., Kollgaard, R. I., Ciardullo, R. et al., 1998, ApJS, 118, 127

Laurent-Muehleisen, S. A., Kollgaard, R., Feigelson, E. D. et al., 1999, ApJ, 525, 127

Ledden, J. E., Odell, S. L., 1985, ApJ, 298, 630

Lewis, L., Eracleous, M., Sambruna, R.M., 2003, ApJ, 593, 115

Lu, H.L, Zhou, H.Y., et al., 2006, AJ, 131, 790

Maraschi, L., et al., 2008, MNRAS, 391, 1981

Marcha, M. J. M., Browne, I. W. A., Impey, C. D et al., 1996, MNRAS, 281, 425

McLure, R. J., Dunlop, J. S. 2004, MNRAS, 352, 1390

McGill, K. L., Woo, J.H., Treu, T. et al., 2008, ApJ, 673, 703

Nagar, N.M., et al., 2000, ApJ, 542, 186

Nagar, N.M., Falcke, H., \& Wilson, A.S., 2005, A\&A, 435, 521

Osterbrock D.E., 1976, PASP, 88, 589

Osterbrock, D. E., Pogge, R. W., 1985, ApJ, 297, 166

Padovani, P., Perlman, E. S., Landt, H. et al., 2003, ApJ, 588, 128

Pei, Y. C., 1992, ApJ, 395, 130

Perlman, E. S., Padovani, P., Giommi, P. et al., 1998, AJ, 115, 1253

Peterson, B. M., 1997, An Introduction to Active Galactic Nuclei(Cambridge: Cambridge Univ.Press)

Piranomonte S., Perri M., Giommi P. et al, 2007, A\&A, 470, 787

Richards, G. T., Lacy, M., Storrie-Lombardi, L. J., et al., 2006, ApJS, 166, 470

Sadler, E.M., Jenkins, C.R., Kotanyi, C.G., 1989, MNRAS, 240, 591

Schlegel, D. J., Finkbeiner, D. P., Davis, M., 1998, ApJ, 500, 525

Stocke, J.T., Morris, S.L., Gioia, I. M., et al, 1991, ApJS, 76, 813

Stoughton, C., Lupton, R. H., Bernardi, M. et al., 2002, AJ, 123, 485

Tadhunter, C.N., et al., 1998, MNRAS, 298, 1035

Tremaine, S., Gebhardt, K., Bender, R. et al., 2002, ApJ, 574, 740

Turriziani, S., Cavazzuti, E., Giommi, P., 2007, A\&A, 472, 699

Urry, C.M. \& Padovani, P., 1995, PASP, 107, 803

Vanden Berk, D. E., et al., 2004, ApJ, 601, 692

Veilleux, S. \& Osterbrock, D. E., 1987, ApJS, 63, 295

Véron-Cetty, M.-P., Joly, M., Véron, P., 2004, A\&A, 417, 515

Vestergaard, M., Peterson, B. M., 2006, ApJ, 641, 689

Voges, W., Aschenbach, B., Boller, Th. et al, 1999, A\&A, 349, 389

Whalen, D. J., Laurent-Muehleisen, S. A. et al, 2006, AJ, 131, 1948

Yuan, W., Zhou, H.Y., \& Komossa, S. et al., 2008, ApJ, 685, 801

Zhou, H.Y., Wang, T.G., Yuan, W.M. et al., 2006, ApJS, 166, 128

Zirbel, E.L., Baum, S.A., 1995, ApJ, 448, 521

This paper was prepared with the RAA LATEX macro v1.2. 